\title{
Fokas Integral Equations for Three Dimensional Layered-Media Scattering
}

\author{
David Ambrose \\ Department of Mathematics \\ Drexel University \\ Philadelphia, PA 19104
}

\author{
David P. Nicholls \\ Department of Mathematics, Statistics, \\ and Computer Science \\ University of Illinois at Chicago \\ Chicago, IL 60607
}

May 19, 2014

\begin{abstract}
The scattering of acoustic waves by periodic structures is of central importance in a wide range of problems of scientific and technological interest. This paper describes a rapid, high-order numerical algorithm for simulating solutions of Helmholtz equations coupled across irregular (non-trivial) interfaces meant to model acoustic waves incident upon a multiply layered medium. Building upon an interfacial formulation from previous work, we describe an Integral Equation strategy inspired by recent developments of Fokas and collaborators for its numerical approximation. The method requires only the discretization of the layer interfaces (so that the number of unknowns is an order of magnitude smaller than volumetric approaches), while it requires neither specialized quadrature rules or periodized fundamental solutions characteristic of many popular Boundary Integral/Element Methods. As with previous contributions by the authors on this formulation, this approach is efficient and spectrally accurate for smooth interfaces.
\end{abstract}

\section{Introduction}

The interaction of acoustic waves with periodic structures plays an important role in many scientific problems. From remote sensing [31] to underwater acoustics [2], the ability to robustly simulate scattered fields with high accuracy is of fundamental importance. Here we focus upon the high-order numerical simulation of solutions of Helmholtz equations coupled across irregular (non-trivial) interfaces meant to model acoustic waves in a multiply layered medium. Based upon a surface formulation recently developed by the author [19], we present a novel Integral Equation Method inspired by recent developments of Fokas and collaborators $[1,9,29,30]$.

Many volumetric numerical algorithms have been devised for the simulation of these problems, for instance, Finite Differences (see, e.g., [26]), Finite Elements (see, e.g., [33]), and Spectral Elements (see, e.g., [13]). These methods suffer from the requirement that they discretize the full volume of the problem domain which results in both a prohibitive number of degrees of freedom, and also the difficult question of appropriately specifying a far-field boundary condition explicitly.

Surface methods are an appealing alternative and those based upon Boundary Integrals (BIM) or Boundary Elements (BEM) are very popular (see, e.g., [28]). In fact, the approach 
we advocate here falls precisely into this category. These BIM/BEM require only discretization of the layer interfaces (rather than the whole structure) and, due to the choice of the Green's function, satisfy the far-field boundary condition exactly. While these methods can deliver high-accuracy simulations with greatly reduced operation counts, there are several difficulties which need to be addressed [27]. First, high-order simulations can only be realized with specially designed quadrature rules which respect the singularities in the Green's function (and its derivative, in certain formulations). Additionally, BIM/BEM typically give rise to dense linear systems to be solved which require carefully designed preconditioned iterative methods (with accelerated matrix-vector products, e.g., by the Fast-Multipole Method [11]) for configurations of engineering interest. Finally, for periodic structures the Green's function must be periodized which greatly increases the computational cost.

Before addressing these concerns as they impact our own formulation, we note that Boundary Perturbation Methods (BPM) have emerged as an appealing strategy which maintain the reduced numbers of degrees of freedom of BIM/BEM while avoiding the need for special quadrature formulas or preconditioned iterative solution procedures for dense systems. Among these are: (i.) the Method of Field Expansions due to Bruno \& Reitich $[3,4,5]$ for doubly layered media, and the generalization of Malcolm \& Nicholls $[15,19]$ to multiply-layered structures; and (ii.) the Method of Operator Expansions due to Milder $[17,18]$ (see also improvements in $[6]$ ) which was generalized to multiple layers by Malcolm \& Nicholls $[14,19]$.

Returning to the challenges faced by BIM/BEM mentioned above, in this contribution we utilize Fokas' approach to discovering Integral Equations (which we term Fokas Integral Equations-FIE) satisfied by the Dirichlet-Neumann Operator (DNO) and its corresponding Dirichlet data. These formulas do not involve the fundamental solution, but rather smooth, "conjugated" solutions of the quasi-periodic Helmholtz problem meaning that simple quadrature rules (e.g., Nyström's Method) may be utilized while periodization is unnecessary. In addition, due to use of a clever alternative to the standard Green's Identity, the derivative of the interface shapes never appear in our FIEs meaning that configurations of rather low smoothness can be accommodated in comparison with alternative approaches (see Appendix A for one choice). The density of the linear systems to be solved cannot be avoided, however, this is somewhat ameliorated by the fact that the number of degrees of freedom required is often quite modest due to the high-order accuracy of our quadratures, and as derivatives of the layer shapes never appear in our integral relations. Finally, the conditioning properties of these FIEs has recently been called into question (see, e.g., the preprint of Wilkening \& Vasan [32]) and, as we discuss in Remark 5.2, they can challenge the effectiveness of such methods. However, for problems of small to moderate size, we have found that the remarkable simplicity and speed of the current algorithm cannot be matched by alternative strategies.

Turning to layered media scattering, we pair these new FIE relationships to the interfacial formulation of such problems recently devised by one of the authors [19]. The resulting algorithm has the speed and efficiency of a boundary method without the complications of iterative linear solvers, Green's function periodization algorithms, or the derivation and implementation of perturbation recursions. One simply builds a linear system of equations with readily computed values and solves with any standard algorithm (e.g., Gaussian elimination).

The rest of the paper is organized as follows: In $\S 2$ we recall the governing equations of layered media scattering, and a surface formulation in $\S 2.1$ (with special cases discussed in $\S 2.2$ ). In $\S 3$ we introduce our new (Fokas) Integral Equations with relations for the top 
layer in $\S 3.1$, the bottom layer in $\S 3.2$, and middle layers in $\S 3.3$ (we summarize these formulas and the zero-perturbation case in $\S 3.4$ ). We discuss formulas for computing the efficiencies in $\S 4$, and numerical results in $\S 5$. We present a class of exact (non-plane-wave) solutions in $\S 5.1$ and numerical implementation and error measurement details in $\S 5.2$. We close with convergence studies in $\S 5.3$ and plane-wave simulations in $\S 5.4$.

\section{Governing Equations}

Consider a $d=\left(d_{1}, d_{2}\right)$-periodic, multiply-layered material with $M$ interfaces at

$$
y=\bar{g}^{(m)}+g^{(m)}\left(x_{1}, x_{2}\right)=\bar{g}^{(m)}+g^{(m)}(x), \quad 1 \leq m \leq M,
$$

where $x=\left(x_{1}, x_{2}\right), \bar{g}^{(m)}$ are constants, and

$$
g^{(m)}(x+d)=g^{(m)}\left(x_{1}+d_{1}, x_{2}+d_{2}\right)=g^{(m)}\left(x_{1}, x_{2}\right)=g^{(m)}(x) .
$$

These interfaces separate $(M+1)$-many layers which define the domains

$$
\begin{array}{rlr}
S^{(0)} & :=\left\{y>\bar{g}^{(1)}+g^{(1)}(x)\right\} & \\
S^{(m)} & :=\left\{\bar{g}^{(m+1)}+g^{(m+1)}(x)<y<\bar{g}^{(m)}+g^{(m)}(x)\right\} & \\
S^{(M)} & :=\left\{y<\bar{g}^{(M)}+g^{(M)}(x)\right\}, &
\end{array}
$$

with (upward pointing) normals $N^{(m)}:=\left(-\nabla_{x} g^{(m)}, 1\right)^{T}$; see Figure 1. In each layer we assume a constant speed $c^{(m)}$ and that the structure is insonified from above by plane-wave incidence

$$
u^{i}(x, y, t)=e^{-i \omega t} e^{i(\alpha \cdot x-\beta y)}=e^{-i \omega t} v^{i}(x, y), \quad \alpha=\left(\alpha_{1}, \alpha_{2}\right)^{T} .
$$

In each layer the quantity $k^{(m)}=\omega / c^{(m)}$ specifies the properties of the material and the frequency of radiation common to the incident and scattered field in the structure. It is wellknown [25] that the problem can be restated as a time-harmonic one of time-independent reduced scattered fields, $v^{(m)}(x, y)$, which, in each layer, are $\alpha$-quasiperiodic

$$
v^{(m)}(x+d, y)=e^{i(\alpha \cdot d)} v^{(m)}(x, y),
$$

and satisfy a Helmholtz equation

$$
\Delta v^{(m)}+\left(k^{(m)}\right)^{2} v^{(m)}=0, \quad \text { in } S^{(m)}, \quad 0 \leq m \leq M .
$$

The reduced fields are coupled through the Dirichlet and Neumann boundary conditions

$$
v^{(m-1)}-v^{(m)}=\zeta^{(m)} \quad y=\bar{g}^{(m)}+g^{(m)}(x), \quad 1 \leq m \leq M,
$$

and

$$
\partial_{N^{(m)}}\left[v^{(m-1)}-v^{(m)}\right]=\psi^{(m)} \quad y=\bar{g}^{(m)}+g^{(m)}(x), \quad 1 \leq m \leq M .
$$

In the case of insonification from above

$$
\begin{array}{rlrl}
\zeta^{(1)} & =v^{i}\left(x, \bar{g}^{(1)}+g^{(1)}(x)\right) & \\
\psi^{(1)} & =\left(\partial_{N^{(1)}} v^{i}\right)\left(x, \bar{g}^{(1)}+g^{(1)}(x)\right) & & \\
\zeta^{(m)} & \equiv 0 & & 2 \leq m \leq M \\
\psi^{(m)} & \equiv 0 & & 2 \leq m \leq M .
\end{array}
$$




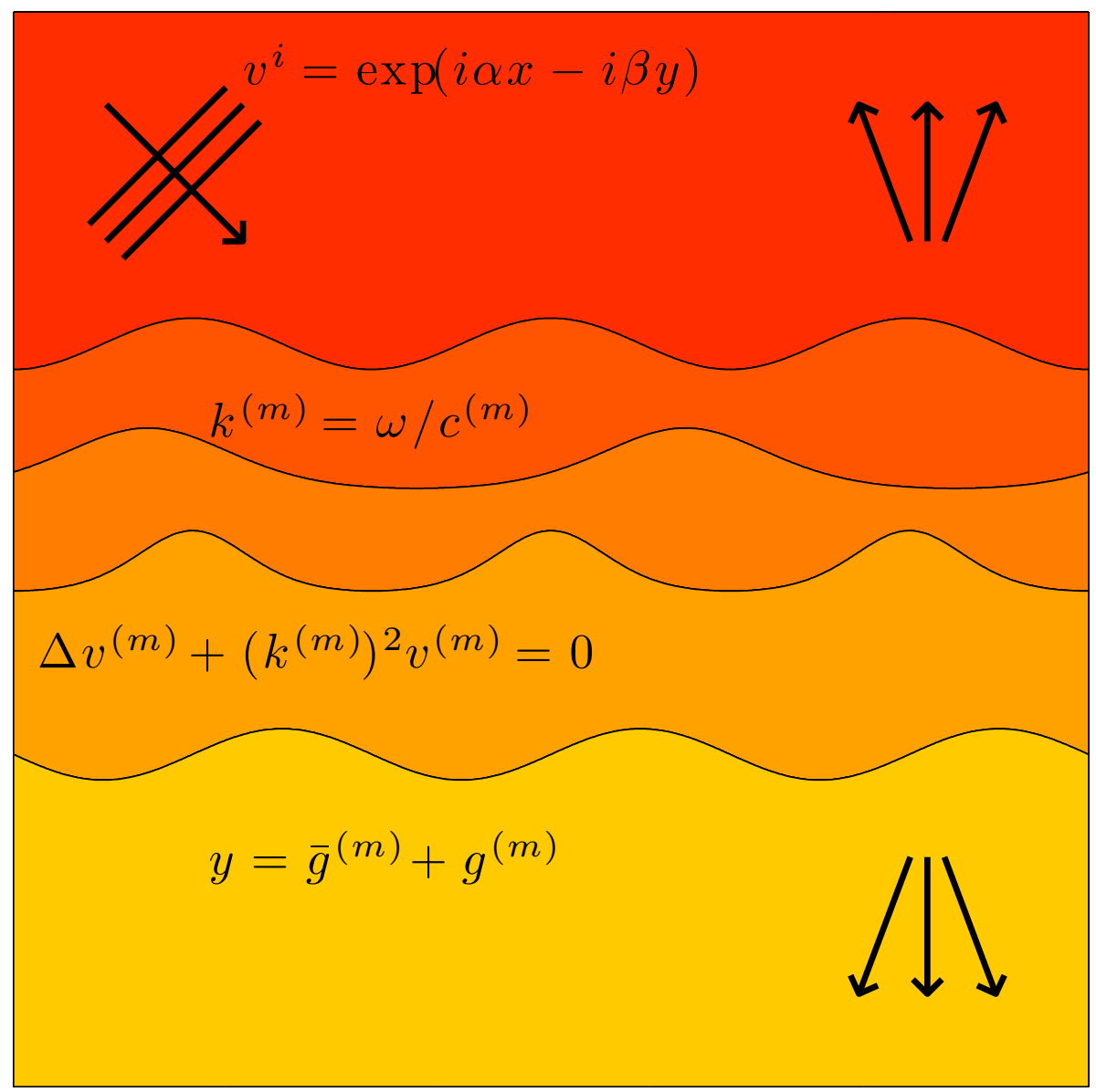

Figure 1: Depiction of a multiply layered media insonified from above by plane-wave radiation.

Finally, outgoing wave conditions are enforced on $v^{(0)}$ and $v^{(M)}$ at positive and negative infinity, respectively.

Remark 2.1. We point out that rather arbitrary incidence can be simulated by the appropriate choice of $\zeta^{(m)}$ and $\psi^{(m)}$. Plane-wave incidence is one of several choices we could make.

\subsection{Boundary Formulation}

We now follow the lead of [19] and restate this problem solely in terms of surface quantities. For this we define the (lower and upper) Dirichlet traces

$$
\begin{aligned}
V^{(m), l}(x) & :=v^{(m)}\left(x, \bar{g}^{(m+1)}+g^{(m+1)}(x)\right) & & 0 \leq m \leq M-1 \\
V^{(m), u}(x) & :=v^{(m)}\left(x, \bar{g}^{(m)}+g^{(m)}(x)\right) & & 1 \leq m \leq M,
\end{aligned}
$$

and the (exterior, lower and upper) Neumann traces

$$
\begin{aligned}
\tilde{V}^{(m), l}(x) & :=-\left(\partial_{N^{(m+1)}} v^{(m)}\right)\left(x, \bar{g}^{(m+1)}+g^{(m+1)}(x)\right) & & 0 \leq m \leq M-1 \\
\tilde{V}^{(m), u}(x) & :=\left(\partial_{N^{(m)}} v^{(m)}\right)\left(x, \bar{g}^{(m)}+g^{(m)}(x)\right) & & 1 \leq m \leq M .
\end{aligned}
$$


With these in hand, the boundary conditions become

$$
\begin{array}{ll}
V^{(m-1), l}-V^{(m), u}=\zeta^{(m)} & 1 \leq m \leq M \\
-\tilde{V}^{(m-1), l}-\tilde{V}^{(m), u}=\psi^{(m)} & 1 \leq m \leq M,
\end{array}
$$

which specifies $(2 M)$ equations for $(4 M)$ unknown functions. This allows us to "eliminate" the upper traces $\left\{\tilde{V}^{(m), u}, V^{(m), u}\right\}$ in favor of the lower ones $\left\{\tilde{V}^{(m), l}, V^{(m), l}\right\}$ by

$$
\begin{aligned}
V^{(m), u} & =V^{(m-1), l}-\zeta^{(m)} & & 1 \leq m \leq M \\
\tilde{V}^{(m), u} & =-\tilde{V}^{(m-1), l}-\psi^{(m)} & & 1 \leq m \leq M .
\end{aligned}
$$

We can generate $(2 M)$ many more equations by defining the "Dirichlet-Neumann Operators" (DNOs)

$$
\begin{aligned}
& G\left[V^{(0), l}\right]:=\tilde{V}^{(0), l} \\
& H(m)\left[V^{(m), u}, V^{(m), l}\right]=\left(\begin{array}{cc}
H^{u u}(m) & H^{u l}(m) \\
H^{l u}(m) & H^{l l}(m)
\end{array}\right)\left[\left(\begin{array}{c}
V^{(m), u} \\
V^{(m), l}
\end{array}\right)\right]:=\left(\begin{array}{c}
\tilde{V}^{(m), u} \\
\tilde{V}^{(m), l}
\end{array}\right) \quad 1 \leq m \leq M-1 \\
& J\left[V^{(M), u}\right]:=\tilde{V}^{(M), u},
\end{aligned}
$$

which relate the Dirichlet quantities, $\left\{V^{(m), u}, V^{(m), l}\right\}$, to the Neumann traces, $\left\{\tilde{V}^{(m), u}, \tilde{V}^{(m), l}\right\}$. From here we diverge from the approach of [19] which described Boundary Perturbation Methods to compute the DNOs $\{G, H(m), J\}$. For the current approach we note that in the following sections we derive integral operators $A$ and $R$ which relate the Dirichlet and Neumann data in the following ways

$$
\begin{aligned}
& A(0) \tilde{V}^{(0), l}-R(0) V^{(0), l}=0 \\
& \left(\begin{array}{cc}
A^{u u}(m) & A^{u l}(m) \\
A^{l u}(m) & A^{l l}(m)
\end{array}\right)\left(\begin{array}{c}
\tilde{V}^{(m), u} \\
\tilde{V}^{(m), l}
\end{array}\right)-\left(\begin{array}{ll}
R^{u u}(m) & R^{u l}(m) \\
R^{l u}(m) & R^{l l}(m)
\end{array}\right)\left(\begin{array}{l}
V^{(m), u} \\
V^{(m), l}
\end{array}\right)=\left(\begin{array}{l}
0 \\
0
\end{array}\right) \quad 1 \leq m \leq M-1 \\
& A(M) \tilde{V}^{(M), u}-R(M) V^{(M), u}=0 .
\end{aligned}
$$

Now, using (2.2), we can write (2.4) as

$$
\begin{gathered}
A(0) \tilde{V}^{(0), l}-R(0) V^{(0), l}=0 \\
\left(\begin{array}{cc}
A^{u u}(m) & A^{u l}(m) \\
A^{l u}(m) & A^{l l}(m)
\end{array}\right)\left(\begin{array}{c}
-\tilde{V}^{(m-1), l}-\psi^{(m)} \\
\tilde{V}^{(m), l}
\end{array}\right)-\left(\begin{array}{cc}
R^{u u}(m) & R^{u l}(m) \\
R^{l u}(m) & R^{l l}(m)
\end{array}\right)\left(\begin{array}{c}
V^{(m-1), l}-\zeta^{(m)} \\
V^{(m), l}
\end{array}\right) \\
=\left(\begin{array}{l}
0 \\
0
\end{array}\right) \quad 1 \leq m \leq M-1 \\
A(M)\left[-\tilde{V}^{(M-1), l}-\psi^{(M)}\right]-R(M)\left[V^{(M-1), l}-\zeta^{(M)}\right]=0 .
\end{gathered}
$$

Simplifying, this can be written as

$$
\mathbf{M V}^{(\mathbf{l})}=\mathbf{Q}
$$


where

$$
\mathbf{M}:=\left(\begin{array}{cccccc}
A(0) & -R(0) & 0 & & \ldots & 0 \\
-A^{u u}(1) & -R^{u u}(1) & A^{u l}(1) & -R^{u l}(1) & \ldots & 0 \\
-A^{l u}(1) & -R^{l u}(1) & A^{l l}(1) & -R^{l l}(1) & \ldots & 0 \\
\vdots & & & & & \vdots \\
0 & \ldots & -A^{u u}(M-1) & -R^{u u}(M-1) & A^{u l}(M-1) & -R^{u l}(M-1) \\
0 & \ldots & -A^{l u}(M-1) & -R^{l u}(M-1) & A^{l l}(M-1) & -R^{l l}(M-1) \\
0 & \ldots & & 0 & -A(M) & -R(M)
\end{array}\right),
$$

and

$$
\mathbf{V}^{(\mathbf{l})}:=\left(\begin{array}{c}
\tilde{V}^{(0), l} \\
V^{(0), l} \\
\vdots \\
\tilde{V}^{(M-1), l} \\
V^{(M-1), l}
\end{array}\right), \quad \mathbf{Q}:=\left(\begin{array}{c}
0 \\
A^{u u}(1) \psi^{(1)}-R^{u u}(1) \zeta^{(1)} \\
A^{l u}(1) \psi^{(1)}-R^{l u}(1) \zeta^{(1)} \\
\vdots \\
A^{u u}(M-1) \psi^{(M-1)}-R^{u u}(M-1) \zeta^{(M-1)} \\
A^{l u}(M-1) \psi^{(M-1)}-R^{l u}(M-1) \zeta^{(M-1)} \\
A(M) \psi^{(M)}-R(M) \zeta^{(M)}
\end{array}\right)
$$

Our numerical method amounts to Nyström's method [8] applied to $\mathbf{M} \mathbf{V}^{(\mathbf{l})}=\mathbf{Q}$ and it only remains to specify the integral operators $A$ and $R$, which we address in $\S 3$.

\section{$2.2 \quad$ Special Cases}

A few special cases of the equations above deserve particular comment and we provide that in this section.

Single Layer. The case of a single layer overlying an impenetrable material does not fit into our framework as stated, however, it can be expanded to include this important configuration. Here, the reduced scattered field, $v^{(0)}$, is still subject to the Helmholtz equation, quasiperiodic boundary conditions, and the outgoing wave condition. Depending upon the properties of the impenetrable layer there is either a Dirichlet boundary condition,

$$
V^{(0), l}(x)=\zeta^{(1)}(x), \quad y=\bar{g}^{(1)}+g^{(1)}(x),
$$

or a Neumann boundary condition,

$$
\tilde{V}^{(0), l}(x)=\psi^{(1)}(x), \quad y=\bar{g}^{(1)}+g^{(1)}(x),
$$

to be enforced at the interface. We can fit into the formulation given above, i.e. solving $\mathbf{M V} \mathbf{V}^{(\mathbf{l})}=\mathbf{Q}$ from (2.5), by making the following choices. For the Dirichlet boundary conditions, (2.6), we set

$$
\mathbf{M}=\left(\begin{array}{cc}
0 & I \\
A(0) & -R(0)
\end{array}\right), \quad \mathbf{V}^{(\mathbf{l})}=\left(\begin{array}{c}
\tilde{V}^{(0), l} \\
V^{(0), l}
\end{array}\right), \quad \mathbf{Q}=\left(\begin{array}{c}
\zeta^{(1)} \\
0
\end{array}\right)
$$

while for the Neumann conditions, (2.7), we equate

$$
\mathbf{M}=\left(\begin{array}{cc}
I & 0 \\
A(0) & -R(0)
\end{array}\right), \quad \mathbf{V}^{(\mathbf{l})}=\left(\begin{array}{c}
\tilde{V}^{(0), l} \\
V^{(0), l}
\end{array}\right), \quad \mathbf{Q}=\left(\begin{array}{c}
\psi^{(1)} \\
0
\end{array}\right)
$$


Remark 2.2. Of course these two could be further simplified to

$$
V^{(0), l}=\zeta^{(1)}, \quad A(0) \tilde{V}^{(0), l}=R(0) \zeta^{(1)},
$$

and

$$
\tilde{V}^{(0), l}=\psi^{(1)}, \quad R(0) V^{(0), l}=A(0) \psi^{(1)},
$$

which requires only the inversion of $A(0)$ or $R(0)$, rather than the full operator $\mathbf{M}$.

Double Layer. For the case of a single layer separating two materials which both permit propagation the boundary conditions become

$$
\begin{array}{ll}
V^{(0), l}-V^{(1), u}=\zeta^{(1)} & y=\bar{g}^{(1)}+g^{(1)}(x) \\
-\tilde{V}^{(0), l}-\tilde{V}^{(1), u}=\psi^{(1)} & y=\bar{g}^{(1)}+g^{(1)}(x),
\end{array}
$$

and we solve $\mathbf{M V} \mathbf{V}^{(\mathbf{l})}=\mathbf{Q}$ with

$$
\mathbf{M}=\left(\begin{array}{cc}
A(0) & -R(0) \\
-A(1) & -R(1)
\end{array}\right), \quad \mathbf{V}^{(\mathbf{l})}=\left(\begin{array}{l}
\tilde{V}^{(0), l} \\
V^{(0), l}
\end{array}\right), \quad \mathbf{Q}=\left(\begin{array}{c}
0 \\
A(1) \psi^{(1)}-R(1) \zeta^{(1)}
\end{array}\right) .
$$

Three Layers. Finally, for a triply layered material we must satisfy the boundary conditions

$$
\begin{array}{ll}
V^{(0), l}-V^{(1), u}=\zeta^{(1)} & y=\bar{g}^{(1)}+g^{(1)}(x) \\
V^{(1), l}-V^{(2), u}=\zeta^{(2)} & y=\bar{g}^{(2)}+g^{(2)}(x) \\
-\tilde{V}^{(0), l}-\tilde{V}^{(1), u}=\psi^{(1)} & y=\bar{g}^{(1)}+g^{(1)}(x) \\
-\tilde{V}^{(1), l}-\tilde{V}^{(2), u}=\psi^{(2)} & y=\bar{g}^{(2)}+g^{(2)}(x),
\end{array}
$$

and we solve $\mathbf{M V}(\mathbf{l})=\mathbf{Q}$ with

$$
\begin{array}{r}
\mathbf{M}=\left(\begin{array}{cccc}
A(0) & -R(0) & 0 & 0 \\
-A^{u u}(1) & -R^{u u}(1) & A^{u l}(1) & -R^{u l}(1) \\
-A^{l u}(1) & -R^{l u}(1) & A^{l l}(1) & -R^{l l}(1) \\
0 & 0 & -A(2) & -R(2)
\end{array}\right), \\
\mathbf{V}^{(\mathbf{l})}=\left(\begin{array}{c}
0 \\
\tilde{V}^{(0), l} \\
V^{(0), l} \\
\tilde{V}^{(1), l} \\
V^{(1), l}
\end{array}\right), \quad \mathbf{Q}=\left(\begin{array}{c}
A^{u u}(1) \psi^{(1)}-R^{u u}(1) \zeta^{(1)} \\
A^{l u}(1) \psi^{(1)}-R^{l u}(1) \zeta^{(1)} \\
A(2) \psi^{(2)}-R(2) \zeta^{(2)}
\end{array}\right) .
\end{array}
$$

Remark 2.3. In the case of plane-wave incidence from above we have

$$
\zeta^{(1)}=e^{i \alpha \cdot x-i \beta\left(\bar{g}^{(1)}+g^{(1)}(x)\right)}, \quad \psi^{(1)}=\left(i \beta+\left(\nabla_{x} g^{(1)}\right) \cdot(i \alpha)\right) e^{i \alpha \cdot x-i \beta\left(\bar{g}^{(1)}+g^{(1)}(x)\right)}
$$

which are the Dirichlet and Neumann traces of the incident field $v^{i}$ at the uppermost boundary $y=\bar{g}^{(1)}+g^{(1)}(x)$. 


\section{Integral Equation Formulation by Fokas' Method}

The reformulation of the Dirichlet-Neumann Operator (DNO) problems we study here come from the remarkable procedure of Fokas $[1,9,29,30]$ which, in our present context, amounts to the inspired use of the following identity which appears in [1].

Lemma 3.1. If we define

$$
Z^{(k)}:=\partial_{y} \phi\left(\Delta \psi+k^{2} \psi\right)+\left(\Delta \phi+k^{2} \phi\right) \partial_{y} \psi
$$

then

$$
Z^{(k)}=\operatorname{div}_{x}\left[F^{(x)}\right]+\partial_{y}\left[F^{(y)}+F^{(k)}\right]
$$

where

$$
F^{(x)}:=\partial_{y} \phi\left(\nabla_{x} \psi\right)+\nabla_{x} \phi\left(\partial_{y} \psi\right), \quad F^{(y)}:=\partial_{y} \phi\left(\partial_{y} \psi\right)-\nabla_{x} \phi \cdot\left(\nabla_{x} \psi\right), \quad F^{(k)}:=k^{2} \phi \psi .
$$

Defining the periodic domain

$$
\begin{aligned}
\Omega=\Omega(\bar{\ell}+\ell(x), \bar{u}+u(x)): & =\{0<x<d\} \times\{\bar{\ell}+\ell(x)<y<\bar{u}+u(x)\}, \\
\ell(x+d) & =\ell(x), \quad u(x+d)=u(x),
\end{aligned}
$$

provided that $\phi$ and $\psi$ solve the Helmholtz equation

$$
\Delta \phi+k^{2} \phi=0, \quad \Delta \psi+k^{2} \psi=0,
$$

then $Z^{(k)}=0$. A (trivial) consequence of the divergence theorem gives us the following Lemma.

Lemma 3.2. Suppose that $\mathbf{G}(x, y)$, defined on $\Omega$, is d-periodic in the $x$ variable, $\mathbf{G}(x+$ $d, y)=\mathbf{G}(x, y)$, where

$$
\mathbf{G}(x, y)=\left(G^{(x)}(x, y), G^{(y)}(x, y)\right)^{T},
$$

then

$$
\int_{\Omega} \operatorname{div}[\mathbf{G}] d V=\int_{0}^{d}\left[G^{(x)} \cdot\left(\nabla_{x} \ell\right)-G^{(y)}\right]_{y=\bar{\ell}+\ell(x)} d x+\int_{0}^{d}\left[-G^{(x)} \cdot\left(\nabla_{x} u\right)+G^{(y)}\right]_{y=\bar{u}+u(x)} d x .
$$

If $\phi$ is $\alpha$-quasiperiodic and $\psi$ is $(-\alpha)$-quasiperiodic, i.e.,

$$
\phi(x+d, y)=e^{i \alpha \cdot d} \phi(x, y), \quad \psi(x+d, y)=e^{-i \alpha \cdot d} \psi(x, y),
$$

then the Lemma 3.2 tells us, with $\mathbf{G}=\left(F^{(x)}, F^{(y)}+F^{(k)}\right)^{T}$,

$$
\begin{aligned}
0=\int_{\Omega} Z^{(k)} d V=\int_{\partial \Omega} \operatorname{div}[\mathbf{G}] d V= & \int_{0}^{d}\left(F^{(x)} \cdot \nabla_{x} \ell-F^{(y)}-F^{(k)}\right)_{y=\bar{\ell}+\ell(x)} d x \\
& +\int_{0}^{d}\left(F^{(x)} \cdot\left(-\nabla_{x} u\right)+F^{(y)}+F^{(k)}\right)_{y=\bar{u}+u(x)} d x,
\end{aligned}
$$


since, in this case, the terms $F^{(x)}, F^{(y)}$, and $F^{(k)}$ are periodic. More specifically,

$$
\begin{aligned}
0=\int_{0}^{d}\left[\partial_{y} \phi\left(\nabla_{x} \psi \cdot \nabla_{x} \ell\right)+\nabla_{x} \phi \cdot\left(\partial_{y} \psi \nabla_{x} \ell\right)\right. & \\
& \left.-\partial_{y} \phi\left(\partial_{y} \psi\right)+\nabla_{x} \phi \cdot\left(\nabla_{x} \psi\right)-k^{2} \phi \psi d x\right]_{y=\bar{\ell}+\ell(x)} \\
+ & \int_{0}^{d}\left[\partial_{y} \phi\left(\nabla_{x} \psi \cdot\left(-\nabla_{x} u\right)\right)+\nabla_{x} \phi \cdot\left(\partial_{y} \psi\left(-\nabla_{x} u\right)\right)\right. \\
& \left.+\partial_{y} \phi\left(\partial_{y} \psi\right)-\nabla_{x} \phi \cdot\left(\nabla_{x} \psi\right)+k^{2} \phi \psi\right]_{y=\bar{u}+u(x)} d x,
\end{aligned}
$$

and

$$
\begin{aligned}
0= & \int_{0}^{d}\left[\partial_{y} \psi\left(\nabla_{x} \ell \cdot \nabla_{x} \phi-\partial_{y} \phi\right)+\nabla_{x} \psi \cdot\left(\nabla_{x} \ell \partial_{y} \phi+\nabla_{x} \phi\right)-\psi k^{2} \phi\right]_{y=\bar{\ell}+\ell(x)} d x \\
& +\int_{0}^{d}\left[\partial_{y} \psi\left(-\nabla_{x} u \cdot \nabla_{x} \phi+\partial_{y} \phi\right)-\nabla_{x} \psi \cdot\left(\nabla_{x} u \partial_{y} \phi+\nabla_{x} \phi\right)+\psi k^{2} \phi\right]_{y=\bar{u}+u(x)} d x
\end{aligned}
$$

If we define

$$
\xi(x):=\phi(x, \bar{\ell}+\ell(x)), \quad \zeta(x):=\phi(x, \bar{u}+u(x)),
$$

then tangential derivatives are given by

$$
\nabla_{x} \xi(x):=\left[\nabla_{x} \phi+\nabla_{x} \ell \partial_{y} \phi\right]_{y=\bar{\ell}+\ell(x)}, \quad \nabla_{x} \zeta(x):=\left[\nabla_{x} \phi+\nabla_{x} u \partial_{y} \phi\right]_{y=\bar{u}+u(x)} .
$$

Recalling the definitions of the DNOs (2.3)

$$
L(x):=\left[-\partial_{y} \phi+\nabla_{x} \ell \cdot \nabla_{x} \phi\right]_{y=\bar{\ell}+\ell(x)}, \quad U(x):=\left[\partial_{y} \phi-\nabla_{x} u \cdot \nabla_{x} \phi\right]_{y=\bar{u}+u(x)},
$$

equation (3.1) now reads

$$
\begin{aligned}
0=\int_{0}^{d}\left(\partial_{y} \psi\right)_{y=\bar{\ell}+\ell(x)} L & +\left(\nabla_{x} \psi\right)_{y=\bar{\ell}+\ell(x)} \cdot \nabla_{x} \xi-(\psi)_{y=\bar{\ell}+\ell(x)} k^{2} \xi d x \\
& +\int_{0}^{d}\left(\partial_{y} \psi\right)_{y=\bar{u}+u(x)} U-\left(\nabla_{x} \psi\right)_{y=\bar{u}+u(x)} \cdot \nabla_{x} \zeta+(\psi)_{y=\bar{u}+u(x)} k^{2} \zeta d x,
\end{aligned}
$$

or

$$
\begin{aligned}
\int_{0}^{d}\left(\partial_{y} \psi\right)_{y=\bar{u}+u(x)} U d x+\int_{0}^{d}\left(\partial_{y} \psi\right)_{y=\bar{\ell}+\ell(x)} L d x & \\
=\int_{0}^{d}\left(\nabla_{x} \psi\right)_{y=\bar{u}+u(x)} & \cdot \nabla_{x} \zeta d x-\int_{0}^{d}\left(\nabla_{x} \psi\right)_{y=\bar{\ell}+\ell(x)} \cdot \nabla_{x} \xi d x \\
& -\int_{0}^{d} k^{2}(\psi)_{y=\bar{u}+u(x)} \zeta d x+\int_{0}^{d} k^{2}(\psi)_{y=\bar{\ell}+\ell(x)} \xi d x
\end{aligned}
$$

\subsection{The Top Layer}

For this problem we consider upward propagating, $\alpha$-quasiperiodic solutions of

$$
\begin{array}{ll}
\Delta \phi+k^{2} \phi=0 & \bar{\ell}+\ell(x)<y<\bar{u} \\
\phi=\xi & y=\bar{\ell}+\ell(x) .
\end{array}
$$


To begin, we note that the Rayleigh expansion [25] gives, for $y>\bar{u}$, that upward propagating $\alpha$-quasiperiodic solutions of the Helmholtz equation can be written as

$$
\phi(x, y)=\sum_{q=-\infty}^{\infty} \hat{\zeta}_{q} e^{i \alpha_{q} \cdot x+i \beta_{q}(y-\bar{u})}, \quad q=\left(q_{1}, q_{2}\right),
$$

where

$$
\alpha_{q}:=\left(\begin{array}{l}
\alpha_{1}+2 \pi q_{1} / d_{1} \\
\alpha_{2}+2 \pi q_{2} / d_{2}
\end{array}\right), \quad \beta_{q}:=\left\{\begin{array}{ll}
\sqrt{k^{2}-\left|\alpha_{q}\right|^{2}} & q \in \mathcal{U} \\
i \sqrt{\left|\alpha_{q}\right|^{2}-k^{2}} & q \notin \mathcal{U}
\end{array},\right.
$$

and the set of propagating modes is specified by

$$
\mathcal{U}:=\left\{\left.q|| \alpha_{q}\right|^{2}<k^{2}\right\} .
$$

Evaluating (3.3) at $y=\bar{u}$ delivers the (generalized) Fourier series of $\zeta(x)$,

$$
\zeta(x)=\sum_{q=-\infty}^{\infty} \hat{\zeta}_{q} e^{i \alpha_{q} \cdot x},
$$

so that we can compute the DNO at $y=\bar{u}$ as

$$
U=\partial_{y} \phi(x, \bar{u})=\sum_{q=-\infty}^{\infty}\left(i \beta_{q}\right) \hat{\zeta}_{q} e^{i \alpha_{q} \cdot x}=:\left(i \beta_{D}\right) \zeta .
$$

Proceeding, we consider an $(-\alpha)$-quasiperiodic "test function"

$$
\psi(x, y)=e^{-i \alpha_{p} \cdot x+i m_{p}(y-\bar{\ell})}
$$

with $m_{p}$ to be determined so that the difference between the first and the sum of the third and fifth terms in (3.2) are zero. For this we consider the quantity

$$
R(x):=\left(\partial_{y} \psi\right)_{y=\bar{u}} U-\left(\nabla_{x} \psi\right)_{y=\bar{u}} \cdot \nabla_{x} \zeta+k^{2}(\psi)_{y=\bar{u}} \zeta
$$

and define

$$
E_{p}:=\exp \left(i m_{p}(\bar{u}-\bar{\ell})\right)
$$

It is easy to show that

$$
\begin{aligned}
R(x) & =\left(i m_{p}\right) e^{-i \alpha_{p} x} E_{p}\left(i \beta_{D}\right) \zeta-\left(-i \alpha_{p}\right) e^{-i \alpha_{p} x} E_{p} \cdot \nabla_{x} \zeta+k^{2} e^{-i \alpha_{p} x} E_{p} \zeta \\
& =\sum_{q=-\infty}^{\infty}\left\{\left(i m_{p}\right)\left(i \beta_{q}\right)-\left(-i \alpha_{p}\right) \cdot\left(i \alpha_{q}\right)+k^{2}\right\} E_{p} \hat{\zeta}_{q} e^{-i\left(\alpha_{p}-\alpha_{q}\right) \cdot x} .
\end{aligned}
$$

Integrating $R$ over the period cell, the only non-zero term features $p=q$ so that

$$
\int_{0}^{d} R(x) d x=|d|\left\{\left(i m_{p}\right)\left(i \beta_{p}\right)-\left(-i \alpha_{p}\right) \cdot\left(i \alpha_{p}\right)+k^{2}\right\} E_{p} \hat{\zeta}_{p} .
$$

Choosing $m_{p}=\beta_{p}$, so that

$$
\psi(x, y)=e^{-i \alpha_{p} x+i \beta_{p}(y-\bar{\ell})},
$$


a "conjugated solution," we get zero since

$$
\alpha_{p} \cdot \alpha_{p}+\beta_{p}^{2}=k^{2} \quad \Longrightarrow \quad\left(i \alpha_{p}\right) \cdot\left(i \alpha_{p}\right)+\left(i \beta_{p}\right)^{2}+k^{2}=0 .
$$

In light of these computations we now have

$$
\int_{0}^{d}\left(\partial_{y} \psi\right)_{y=\bar{\ell}+\ell(x)} L d x=-\int_{0}^{d}\left(\nabla_{x} \psi\right)_{y=\bar{\ell}+\ell(x)} \cdot \nabla_{x} \xi d x+\int_{0}^{d} k^{2}(\psi)_{y=\bar{\ell}+\ell(x)} \xi d x
$$

and, with $\psi$ defined above,

$$
\int_{0}^{d}\left(i \beta_{p}\right) e^{i \beta_{p} \ell} e^{-i \alpha_{p} x} L d x=-\int_{0}^{d}\left(-i \alpha_{p}\right) e^{i \beta_{p} \ell} e^{-i \alpha_{p} x} \cdot \nabla_{x} \xi d x+\int_{0}^{d} k^{2} e^{i \beta_{p} \ell} e^{-i \alpha_{p} x} \xi d x .
$$

To match with $(2.3)$ we rename the DNO $G$ and the interface $g$ giving

$$
\int_{0}^{d}\left(i \beta_{p}\right) e^{i \beta_{p} g} e^{-i \alpha_{p} x} G d x=\int_{0}^{d}\left(i \alpha_{p}\right) e^{i \beta_{p} g} e^{-i \alpha_{p} x} \cdot \nabla_{x} \xi d x+\int_{0}^{d} k^{2} e^{i \beta_{p} g} e^{-i \alpha_{p} x} \xi d x .
$$

Remark 3.3. At this point one can ask how the current procedure differs from that of [1]. Here we have chosen $\psi$ at the outset so that $\int R=0$. In contrast, [1] chose two $(-\alpha)-$ quasiperiodic solutions and then combined them in such a way that these "far field" terms disappeared. We also point out that we follow the developments of [1] quite closely which is not the same as the "method" devised and analyzed in $[9,29,30]$ (which we note does not deliver formulas for the three-dimensional problem).

\subsection{The Bottom Layer}

In a similar fashion we can consider downward propagating, $\alpha$-quasiperiodic solutions of

$$
\begin{array}{ll}
\Delta \phi+k^{2} \phi=0 & \bar{\ell}<y<\bar{u}+u(x) \\
\phi=\zeta & y=\bar{u}+u(x),
\end{array}
$$

and the "test function"

$$
\psi(x, y)=e^{-i \alpha_{p} x-i \beta_{p}(y-\bar{u})} .
$$

With this choice of $\psi$ the second, fourth, and sixth terms in (3.2) combine to zero and we find

$$
\int_{0}^{d}\left(\partial_{y} \psi\right)_{y=\bar{u}+u(x)} U d x=\int_{0}^{d}\left(\nabla_{x} \psi\right)_{y=\bar{u}+u(x)} \cdot \nabla_{x} \zeta d x-\int_{0}^{d} k^{2}(\psi)_{y=\bar{u}+u(x)} \zeta d x
$$

With $\psi$ defined in this way we determine that

$$
\int_{0}^{d}\left(-i \beta_{p}\right) e^{-i \beta_{p} u} e^{-i \alpha_{p} x} U d x=\int_{0}^{d}\left(-i \alpha_{p}\right) e^{-i \beta_{p} u} e^{-i \alpha_{p} x} \cdot \nabla_{x} \zeta d x-\int_{0}^{d} k^{2} e^{-i \beta_{p} u} e^{-i \alpha_{p} x} \zeta d x .
$$

Again, to match with $(2.3)$ we rename the DNO $J$ and the interface $g$ giving

$$
\int_{0}^{d}\left(i \beta_{p}\right) e^{-i \beta_{p} g} e^{-i \alpha_{p} x} J d x=\int_{0}^{d}\left(i \alpha_{p}\right) e^{-i \beta_{p} g} e^{-i \alpha_{p} x} \cdot \nabla_{x} \zeta d x+\int_{0}^{d} k^{2} e^{-i \beta_{p} g} e^{-i \alpha_{p} x} \zeta d x .
$$




\subsection{A Middle Layer}

Finally, we consider $\alpha$-quasiperiodic solutions of

$$
\begin{array}{ll}
\Delta \phi+k^{2} \phi=0 & \bar{\ell}+\ell(x)<y<\bar{u}+u(x) \\
\phi=\xi & y=\bar{\ell}+\ell(x) \\
\phi=\zeta & y=\bar{u}+u(x),
\end{array}
$$

and the "test functions"

$$
\begin{aligned}
\psi^{(u)}(x, y) & =\frac{\cosh \left(i \beta_{p}(y-\bar{\ell})\right)}{\sinh \left(i \beta_{p}(\bar{u}-\bar{\ell})\right)} e^{-i \alpha_{p} x} \\
\psi^{(\ell)}(x, y) & =\frac{\cosh \left(i \beta_{p}(\bar{u}-y)\right)}{\sinh \left(i \beta_{p}(\bar{u}-\bar{\ell})\right)} e^{-i \alpha_{p} x} .
\end{aligned}
$$

Defining

$$
\begin{array}{cl}
\operatorname{co}_{p}:=\operatorname{coth}\left(i \beta_{p}(\bar{u}-\bar{\ell})\right), & \operatorname{cs}_{p}:=\operatorname{csch}\left(i \beta_{p}(\bar{u}-\bar{\ell})\right), \\
C(u):=\cosh \left(i \beta_{p} u\right), & S(u):=\sinh \left(i \beta_{p} u\right), \\
C(\ell):=\cosh \left(i \beta_{p} \ell\right), & S(\ell):=\sinh \left(i \beta_{p} \ell\right),
\end{array}
$$

we can show that

$$
\begin{aligned}
\psi^{(u)}(x, \bar{u}+u) & =\left(\operatorname{co}_{p} C(u)+S(u)\right) e^{-i \alpha_{p} x} \\
\psi^{(u)}(x, \bar{\ell}+\ell) & =\operatorname{cs}_{p} C(\ell) e^{-i \alpha_{p} x} \\
\psi^{(\ell)}(x, \bar{u}+u) & =\operatorname{cs}_{p} C(u) e^{-i \alpha_{p} x} \\
\psi^{(\ell)}(x, \bar{\ell}+\ell) & =\left(\operatorname{co}_{p} C(\ell)-S(\ell)\right) e^{-i \alpha_{p} x},
\end{aligned}
$$

and

$$
\begin{aligned}
\partial_{x} \psi^{(u)}(x, \bar{u}+u) & =\left(-i \alpha_{p}\right)\left(\operatorname{co}_{p} C(u)+S(u)\right) e^{-i \alpha_{p} x} \\
\partial_{x} \psi^{(u)}(x, \bar{\ell}+\ell) & =\left(-i \alpha_{p}\right) \operatorname{cs}_{p} C(\ell) e^{-i \alpha_{p} x} \\
\partial_{x} \psi^{(\ell)}(x, \bar{u}+u) & =\left(-i \alpha_{p}\right) \operatorname{cs}_{p} C(u) e^{-i \alpha_{p} x} \\
\partial_{x} \psi^{(\ell)}(x, \bar{\ell}+\ell) & =\left(-i \alpha_{p}\right)\left(\operatorname{co}_{p} C(\ell)-S(\ell)\right) e^{-i \alpha_{p} x},
\end{aligned}
$$

and

$$
\begin{aligned}
\partial_{y} \psi^{(u)}(x, \bar{u}+u) & =\left(i \beta_{p}\right)\left(C(u)+\operatorname{co}_{p} S(u)\right) e^{-i \alpha_{p} x} \\
\partial_{y} \psi^{(u)}(x, \bar{\ell}+\ell) & =\left(i \beta_{p}\right) \operatorname{cs}_{p} S(\ell) e^{-i \alpha_{p} x} \\
\partial_{y} \psi^{(\ell)}(x, \bar{u}+u) & =\left(i \beta_{p}\right) \operatorname{cs}_{p} S(u) e^{-i \alpha_{p} x} \\
\partial_{y} \psi^{(\ell)}(x, \bar{\ell}+\ell) & =\left(-i \beta_{p}\right)\left(C(\ell)-\operatorname{co}_{p} S(\ell)\right) e^{-i \alpha_{p} x} .
\end{aligned}
$$

From (3.2), with $\psi^{(u)}$ we find

$$
\begin{aligned}
& \int_{0}^{d}\left(i \beta_{p}\right)(C(u)+\left.\operatorname{co}_{p} S(u)\right) e^{-i \alpha_{p} x} U d x+\int_{0}^{d}\left(i \beta_{p}\right) \operatorname{cs}_{p} S(\ell) e^{-i \alpha_{p} x} L d x \\
&=\int_{0}^{d}\left(-i \alpha_{p}\right)\left(\operatorname{co}_{p} C(u)+S(u)\right) e^{-i \alpha_{p} x} \cdot \nabla_{x} \zeta d x-\int_{0}^{d}\left(-i \alpha_{p}\right) \operatorname{cs}_{p} C(\ell) e^{-i \alpha_{p} x} \cdot \nabla_{x} \xi d x \\
&-\int_{0}^{d} k^{2}\left(\operatorname{co}_{p} C(u)+S(u)\right) e^{-i \alpha_{p} x} \zeta d x+\int_{0}^{d} k^{2} \operatorname{cs}_{p} C(\ell) e^{-i \alpha_{p} x} \xi d x .
\end{aligned}
$$


Additionally, with $\psi^{(\ell)},(3.2)$ delivers,

$$
\begin{aligned}
\int_{0}^{d}\left(i \beta_{p}\right) \operatorname{cs}_{p} S(u) e^{-i \alpha_{p} x} U d x+\int_{0}^{d}\left(-i \beta_{p}\right)\left(C(\ell)-\operatorname{co}_{p} S(\ell)\right) e^{-i \alpha_{p} x} L d x \\
=\int_{0}^{d}\left(-i \alpha_{p}\right) \operatorname{cs}_{p} C(u) e^{-i \alpha_{p} x} \cdot \nabla_{x} \zeta d x-\int_{0}^{d}\left(-i \alpha_{p}\right)\left(\operatorname{co}_{p} C(\ell)-S(\ell)\right) e^{-i \alpha_{p} x} \cdot \nabla_{x} \xi d x \\
-\int_{0}^{d} k^{2} \operatorname{cs}_{p} C(u) e^{-i \alpha_{p} x} \zeta d x+\int_{0}^{d} k^{2}\left(\cos _{p} C(\ell)-S(\ell)\right) e^{-i \alpha_{p} x} \xi d x
\end{aligned}
$$

\subsection{Summary of Formulas and Zero-Deformation Simplifications}

We point out that all of the formulas derived thus far, (3.5), (3.6), (3.7), and (3.8), can be stated generically as

$$
\hat{A}_{p}[\tilde{V}]=\hat{R}_{p}[V]
$$

1. (Top Layer) For (3.5), after dividing by $\left(i \beta_{p}\right)$,

$$
\begin{aligned}
& \tilde{V}=G, \quad V=\xi, \quad \hat{A}_{p}(g)[G]=\int_{0}^{d} e^{i \beta_{p} g} e^{-i \alpha_{p} x} G(x) d x \\
& \hat{R}_{p}(g)[\xi]=\int_{0}^{d} e^{i \beta_{p} g} e^{-i \alpha_{p} x}\left\{\frac{i \alpha_{p}}{i \beta_{p}} \cdot \nabla_{x}+\frac{k^{2}}{i \beta_{p}}\right\} \xi(x) d x .
\end{aligned}
$$

2. (Bottom Layer) For (3.6), after dividing by $\left(i \beta_{p}\right)$,

$$
\begin{aligned}
& \tilde{V}=J, \quad V=\zeta, \quad \hat{A}_{p}(g)[J]=\int_{0}^{d} e^{-i \beta_{p} g} e^{-i \alpha_{p} x} J(x) d x \\
& \hat{R}_{p}(g)[\zeta]=\int_{0}^{d} e^{-i \beta_{p} g} e^{-i \alpha_{p} x}\left\{\frac{i \alpha_{p}}{i \beta_{p}} \cdot \nabla_{x}+\frac{k^{2}}{i \beta_{p}}\right\} \zeta(x) d x .
\end{aligned}
$$

3. For (3.7) \& (3.8), after dividing by $\left(i \beta_{p}\right)$,

$$
\begin{aligned}
\tilde{V} & =\left(\begin{array}{c}
\tilde{V}^{u} \\
\tilde{V}^{\ell}
\end{array}\right)=\left(\begin{array}{l}
U \\
L
\end{array}\right), \quad V=\left(\begin{array}{l}
V^{u} \\
V^{\ell}
\end{array}\right)=\left(\begin{array}{l}
\zeta \\
\xi
\end{array}\right) \\
\hat{A}_{p}(u, \ell)\left[\left(\begin{array}{l}
U \\
L
\end{array}\right)\right]=\int_{0}^{d} & \left(\begin{array}{cc}
C(u)+\operatorname{co}_{p} S(u) & \operatorname{cs}_{p} S(\ell) \\
-\operatorname{cs}_{p} S(u) & C(\ell)-\operatorname{co}_{p} S(\ell)
\end{array}\right)\left(\begin{array}{l}
U \\
L
\end{array}\right) e^{-i \alpha_{p} x} d x \\
\hat{R}_{p}(u, \ell)\left[\left(\begin{array}{l}
\zeta \\
\xi
\end{array}\right)\right]= & \int_{0}^{d}\left(\begin{array}{cc}
-\operatorname{co}_{p} C(u)-S(u) & \operatorname{cs}_{p} C(\ell) \\
\operatorname{cs}_{p} C(u) & -\operatorname{co}_{p} C(\ell)+S(\ell)
\end{array}\right) \\
& \times\left\{\frac{i \alpha_{p}}{i \beta_{p}} \cdot \nabla_{x}+\frac{k^{2}}{i \beta_{p}}\right\}\left(\begin{array}{l}
\zeta \\
\xi
\end{array}\right) e^{-i \alpha_{p} x} d x
\end{aligned}
$$

In the class of flat interfaces $(g \equiv 0, u \equiv 0, \ell \equiv 0)$ we have

1. (Top Layer)

$$
\begin{gathered}
\hat{A}_{p}(0)[G]=\int_{0}^{d} e^{-i \alpha_{p} x} G(x) d x \\
\hat{R}_{p}(0)[\xi]=\int_{0}^{d} e^{-i \alpha_{p} x}\left\{\frac{i \alpha_{p}}{i \beta_{p}} \cdot \nabla_{x}+\frac{k^{2}}{i \beta_{p}}\right\} \xi(x) d x .
\end{gathered}
$$


2. (Bottom Layer)

$$
\begin{gathered}
\hat{A}_{p}(0)[J]=\int_{0}^{d} e^{-i \alpha_{p} x} J(x) d x \\
\hat{R}_{p}(0)[\zeta]=\int_{0}^{d} e^{-i \alpha_{p} x}\left\{\frac{i \alpha_{p}}{i \beta_{p}} \cdot \nabla_{x}+\frac{k^{2}}{i \beta_{p}}\right\} \zeta(x) d x .
\end{gathered}
$$

3. (Middle Layer)

$$
\begin{gathered}
\hat{A}_{p}(0,0)\left[\left(\begin{array}{c}
U \\
L
\end{array}\right)\right]=\int_{0}^{d}\left(\begin{array}{ll}
1 & 0 \\
0 & 1
\end{array}\right)\left(\begin{array}{l}
U \\
L
\end{array}\right) e^{-i \alpha_{p} x} d x \\
\hat{R}_{p}(0,0)\left[\left(\begin{array}{l}
\zeta \\
\xi
\end{array}\right)\right]=\int_{0}^{d}\left(\begin{array}{cc}
-\operatorname{co}_{p} & \operatorname{cs}_{p} \\
\operatorname{cs}_{p} & -\operatorname{co}_{p}
\end{array}\right)\left\{\frac{i \alpha_{p}}{i \beta_{p}} \cdot \nabla_{x}+\frac{k^{2}}{i \beta_{p}}\right\}\left(\begin{array}{l}
\zeta \\
\xi
\end{array}\right) e^{-i \alpha_{p} x} d x .
\end{gathered}
$$

Recognizing the Fourier transform

$$
\hat{\psi}_{p}=F[\psi]=\int_{0}^{d} e^{-i \alpha_{p} x} \psi(x) d x
$$

and using the fact that $\left(i \alpha_{p}\right) \cdot\left(i \alpha_{p}\right)+k^{2}=-\left(i \beta_{p}\right)^{2}$ we find

1. (Top Layer)

$$
\begin{gathered}
\hat{A}_{p}(0)[G]=\hat{G}_{p} \\
\hat{R}_{p}(0)[\xi]=\left\{\frac{i \alpha_{p}}{i \beta_{p}} \cdot\left(i \alpha_{p}\right)+\frac{k^{2}}{i \beta_{p}}\right\} \hat{\xi}_{p}=-\left(i \beta_{p}\right) \hat{\xi}_{p} .
\end{gathered}
$$

2. (Bottom Layer)

$$
\begin{gathered}
\hat{A}_{p}(0)[J]=\hat{J}_{p} \\
\hat{R}_{p}(0)[\zeta]=\left\{\frac{i \alpha_{p}}{i \beta_{p}} \cdot\left(i \alpha_{p}\right)+\frac{k^{2}}{i \beta_{p}}\right\} \hat{\zeta}_{p}=-\left(i \beta_{p}\right) \hat{\zeta}_{p} .
\end{gathered}
$$

3. (Middle Layer)

$$
\begin{gathered}
\hat{A}_{p}(0,0)\left[\left(\begin{array}{l}
U \\
L
\end{array}\right)\right]=\left(\begin{array}{ll}
1 & 0 \\
0 & 1
\end{array}\right)\left(\begin{array}{l}
\hat{U}_{p} \\
\hat{L}_{p}
\end{array}\right) \\
\hat{R}_{p}(0,0)\left[\left(\begin{array}{l}
\zeta \\
\xi
\end{array}\right)\right]=\left(\begin{array}{cc}
-\operatorname{co}_{p} & \operatorname{cs}_{p} \\
\operatorname{cs}_{p} & -\operatorname{co}_{p}
\end{array}\right)\left\{\frac{i \alpha_{p}}{i \beta_{p}} \cdot\left(i \alpha_{p}\right)+\frac{k^{2}}{i \beta_{p}}\right\}\left(\begin{array}{c}
\hat{\zeta}_{p} \\
\hat{\xi}_{p}
\end{array}\right) \\
=\left(\begin{array}{cc}
-\operatorname{co}_{p} & \operatorname{cs}_{p} \\
\operatorname{cs}_{p} & -\mathrm{co}_{p}
\end{array}\right)\left(-i \beta_{p}\right)\left(\begin{array}{l}
\hat{\zeta}_{p} \\
\hat{\xi}_{p}
\end{array}\right),
\end{gathered}
$$

and discover the classical results

$$
\begin{gathered}
\hat{G}_{p}=-\left(i \beta_{p}\right) \hat{\xi}_{p}, \quad \hat{J}_{p}=-\left(i \beta_{p}\right) \hat{\zeta}_{p} \\
\left(\begin{array}{c}
\hat{U}_{p} \\
\hat{L}_{p}
\end{array}\right)=\left(i \beta_{p}\right)\left(\begin{array}{cc}
\operatorname{co}_{p} & -\operatorname{cs}_{p} \\
-\operatorname{cs}_{p} & \operatorname{co}_{p}
\end{array}\right)\left(\begin{array}{l}
\hat{\zeta}_{p} \\
\hat{\xi}_{p}
\end{array}\right) .
\end{gathered}
$$


We close by pointing out that (3.9) specifies equations for the Fourier coefficients of $\tilde{V}$ and $V$ rather than the functions themselves. To specify equations for the latter, as a function of the variable $\tilde{x}$, we simply invert the Fourier transform, e.g.,

$$
A[\tilde{V}]=R[V]
$$

where

$$
A=\frac{1}{|d|} \sum_{p=-\infty}^{\infty} \hat{A}_{p} e^{i \alpha_{p} \cdot \tilde{x}}, \quad R=\frac{1}{|d|} \sum_{p=-\infty}^{\infty} \hat{R}_{p} e^{i \alpha_{p} \cdot \tilde{x}} .
$$

In our simulations below we apply Nyström's method [8] to (3.13) instead of (3.9).

Remark 3.4. Due to the smoothness of solutions in the presence of smooth interfaces, we choose equally spaced gridpoints in our Nyström approach resulting in the trapezoidal rule. We remark that Fast Fourier Transforms (FFTs) [10] could be used as a fast inversion strategy for this flat-interface configuration. However, such an approach cannot be used for general deformations.

\section{Computing Far-Field Information: The Efficiencies}

In many situations it is insufficient to know the scattered fields at the layer interfaces, for instance when "far field" data is required. In periodic layered media scattering, such information is encoded in the efficiencies [25] and in this section we describe how the Fokas formalism can be used to derive equations for these from the unknowns of the problem.

To begin we once again use the Rayleigh expansions which state that above the structure the scattered field can be expressed as

$$
v^{(0)}(x, y)=\sum_{p=-\infty}^{\infty} B_{p}^{(0)} e^{i \alpha_{p} \cdot x+i \beta_{p}^{(0)} y},
$$

while below the structure

$$
v^{(M)}(x, y)=\sum_{p=-\infty}^{\infty} B_{p}^{(M)} e^{i \alpha_{p} \cdot x-i \beta_{p}^{(M)} y} .
$$

The upper and lower efficiencies (together with the set of propagating modes) are defined by

$$
\begin{aligned}
e_{p}^{(0)}:=\frac{\beta_{p}^{(0)}}{\beta}\left|B_{p}^{(0)}\right|^{2}, & p \in \mathcal{U}^{(0)}=\left\{\left.p|| \alpha_{p}\right|^{2}<\left(k^{(0)}\right)^{2}\right\} \\
e_{p}^{(M)}:=\frac{\beta_{p}^{(M)}}{\beta}\left|B_{p}^{(M)}\right|^{2}, & p \in \mathcal{U}^{(M)}=\left\{\left.p|| \alpha_{p}\right|^{2}<\left(k^{(M)}\right)^{2}\right\} .
\end{aligned}
$$

There is a principle of conservation of energy for lossless media which states that

$$
\sum_{p \in \mathcal{U}^{(0)}} e_{p}^{(0)}+\sum_{p \in \mathcal{U}^{(M)}} e_{p}^{(M)}=1
$$

which gives a diagnostic of convergence, the "energy defect"

$$
\delta:=1-\sum_{p \in \mathcal{U}^{(0)}} e_{p}^{(0)}-\sum_{p \in \mathcal{U}^{(M)}} e_{p}^{(M)} .
$$


We now seek formulae to recover the $\left\{B_{p}^{(0)}, B_{p}^{(M)}\right\}$ from the Dirichlet and Neumann traces which we can compute from our algorithm. We begin with the uppermost layer and, for simplicity, drop the zero-superscript. Consider the hyperplane $y=\bar{u}\left(\bar{u}>\bar{g}^{(1)}+g^{(1)}(x)\right)$ and the Dirichlet trace

$$
\zeta(x):=v(x, \bar{u})=\sum_{p=-\infty}^{\infty} B_{p} e^{i \alpha_{p} \cdot x+i \beta_{p} \bar{u}} .
$$

Therefore, if we can recover $\zeta(x)$ then

$$
\sum_{p=-\infty}^{\infty} \hat{\zeta}_{p} e^{i \alpha_{p} \cdot x}=\zeta(x)=v(x, \bar{u})=\sum_{p=-\infty}^{\infty} B_{p} e^{i \alpha_{p} \cdot x+i \beta_{p} \bar{u}}
$$

which gives

$$
B_{p}=\hat{\zeta}_{p} e^{-i \beta_{p} \bar{u}}
$$

Once again, we work with (3.2) and recall that in this flat-interface case, c.f. (3.4),

$$
U=\left(i \beta_{D}\right) \zeta
$$

We suppose that we know the following data at $y=\bar{\ell}+\ell(x)$ :

$$
\xi(x), \quad \nabla_{x} \xi(x), \quad L(x)
$$

and, in the same spirit as $\S 3.1$, seek a relation between these and $\zeta$. Of course, if we utilize the same function $\psi$ then the data at $y=\bar{u}$ disappear entirely, however, if we change this slightly (effectively a change of sign in the $y$-dependence) to

$$
\psi(x, y)=e^{-i \alpha_{p} \cdot x+i \beta_{p}(\bar{u}-y)},
$$

we can realize a convenient formula for $\hat{\zeta}_{p}$. We insert this choice into (3.2) to deliver

$$
\begin{gathered}
\int_{0}^{d}\left(-i \beta_{p}\right) e^{-i \alpha_{p} x} U d x+\int_{0}^{d}\left(-i \beta_{p}\right) e^{i \beta_{p}(\bar{u}-\bar{\ell})} e^{-i \beta_{p} \ell(x)} e^{-i \alpha_{p} x} L d x \\
=\int_{0}^{d}\left(-i \alpha_{p}\right) e^{-i \alpha_{p} x} \cdot \nabla_{x} \zeta d x-\int_{0}^{d}\left(-i \alpha_{p}\right) e^{i \beta_{p}(\bar{u}-\bar{\ell})} e^{-i \beta_{p} \ell(x)} e^{-i \alpha_{p} x} \cdot \nabla_{x} \xi d x \\
\quad-\int_{0}^{d} k^{2} e^{-i \alpha_{p} x} \zeta d x+\int_{0}^{d} k^{2} e^{i \beta_{p}(\bar{u}-\bar{\ell})} e^{-i \beta_{p} \ell(x)} e^{-i \alpha_{p} x} \xi d x .
\end{gathered}
$$

Moving the data at $y=\bar{u}$ to the left and terms evaluated at $y=\bar{\ell}+\ell(x)$ to the right, and, once again, recognizing the Fourier transforms, we find

$$
\left[\left(-i \beta_{p}\right)\left(i \beta_{p}\right)+\left(i \alpha_{p}\right) \cdot\left(i \alpha_{p}\right)+k^{2}\right] \hat{\zeta}_{p}=\hat{Q}_{p}
$$

where

$$
Q(x)=e^{i \beta_{p}(\bar{u}-\bar{\ell})} e^{-i \beta_{p} \ell(x)}\left\{\left(i \beta_{p}\right) L(x)+\left(i \alpha_{p}\right) \cdot \nabla_{x} \xi(x)+k^{2} \xi(x)\right\} .
$$

We can simplify this to

$$
\hat{\zeta}_{p}=\frac{\hat{Q}_{p}}{\left[-2\left(i \beta_{p}\right)^{2}\right]}
$$


which delivers

$$
B_{p}=-e^{-i \beta_{p} \bar{u}} \frac{\hat{Q}_{p}}{2\left(i \beta_{p}\right)^{2}}
$$

In the simple case of a flat lower interface, $\ell(x) \equiv 0$, we find

$$
Q(x)=e^{i \beta_{p}(\bar{u}-\bar{\ell})}\left\{\left(i \beta_{p}\right) L(x)+\left(i \alpha_{p}\right) \cdot \nabla_{x} \xi(x)+k^{2} \xi(x)\right\},
$$

so

$$
\hat{Q}_{p}=e^{i \beta_{p}(\bar{u}-\bar{\ell})}\left\{\left(i \beta_{p}\right) \hat{L}_{p}+\left(i \alpha_{p}\right) \cdot\left(i \alpha_{p}\right) \hat{\xi}_{p}+k^{2} \hat{\xi}_{p}\right\}=e^{i \beta_{p}(\bar{u}-\bar{\ell})}\left\{-2\left(i \beta_{p}\right)^{2}\right\}
$$

and

$$
B_{p}=-e^{-i \beta_{p} \bar{u}} \frac{\hat{Q}_{p}}{2\left(i \beta_{p}\right)^{2}}=-e^{-i \beta_{p} \bar{u}} \frac{1}{2\left(i \beta_{p}\right)^{2}} e^{i \beta_{p}(\bar{u}-\bar{\ell})}\left(-2\left(i \beta_{p}\right)^{2}\right)=e^{-i \beta_{p} \bar{\ell}} .
$$

For the lower-layer Rayleigh coefficients we can proceed in much the same way. Here we drop the $(M)$-superscript and denote the Rayleigh coefficients by $C_{p}$. Consider $y=\bar{\ell}$ $\left(\bar{\ell}<\bar{g}_{M}+g_{M}(x)\right)$ and the Dirichlet trace

$$
\xi(x):=v(x, \bar{\ell})=\sum_{p=-\infty}^{\infty} C_{p} e^{i \alpha_{p} \cdot x-i \beta_{p} \bar{\ell}} .
$$

Therefore, if we can recover $\xi(x)$ then

$$
\sum_{p=-\infty}^{\infty} \hat{\xi}_{p} e^{i \alpha_{p} \cdot x}=\xi(x)=v(x, \bar{\ell})=\sum_{p=-\infty}^{\infty} C_{p} e^{i \alpha_{p} \cdot x-i \beta_{p} \bar{\ell}}
$$

which gives

$$
C_{p}=\hat{\xi}_{p} e^{i \beta_{p} \bar{\ell}}
$$

If we now follow $\S 3.2$ with $\ell(x) \equiv 0$ (noting that $L=-\left(-i \beta_{D}\right)=\left(i \beta_{D}\right)$ ), but now choose

$$
\psi(x, y)=e^{-i \alpha_{p} x+i \beta_{p}(y-\bar{\ell})}
$$

then (3.2) gives

$$
\begin{aligned}
& \int_{0}^{d}\left(i \beta_{p}\right) e^{i \beta_{p}(\bar{u}-\bar{\ell})} e^{i \beta_{p} u(x)} e^{-i \alpha_{p} x} U d x+\int_{0}^{d}\left(i \beta_{p}\right) e^{-i \alpha_{p} x} L d x \\
&=\int_{0}^{d}\left(-i \alpha_{p}\right) e^{i \beta_{p}(\bar{u}-\bar{\ell})} e^{i \beta_{p} u(x)} e^{-i \alpha_{p} x} \cdot \nabla_{x} \zeta d x-\int_{0}^{d}\left(-i \alpha_{p}\right) e^{-i \alpha_{p} x} \cdot \nabla_{x} \xi d x \\
&-\int_{0}^{d} k^{2} e^{i \beta_{p}(\bar{u}-\bar{\ell})} e^{i \beta_{p} u(x)} e^{-i \alpha_{p} x} \zeta d x+\int_{0}^{d} k^{2} e^{-i \alpha_{p} x} \xi d x .
\end{aligned}
$$

Now, moving the data at $y=\bar{\ell}$ to the left and the terms at $y=\bar{u}+u(x)$ to the right, we recognize the Fourier transform

$$
\left[\left(i \beta_{p}\right)\left(i \beta_{p}\right)-\left(i \alpha_{p}\right) \cdot\left(i \alpha_{p}\right)-k^{2}\right] \hat{\xi}_{p}=\hat{R}_{p}
$$

where

$$
R(x)=e^{i \beta_{p}(\bar{u}-\bar{\ell})} e^{i \beta_{p} u(x)}\left\{-\left(i \beta_{p}\right) U(x)-\left(i \alpha_{p}\right) \cdot \nabla_{x} \zeta(x)-k^{2} \zeta(x)\right\}
$$


We can simplify this to

$$
\hat{\xi}_{p}=\frac{\hat{R}_{p}}{\left[2\left(i \beta_{p}\right)^{2}\right]}
$$

which delivers

$$
C_{p}=e^{i \beta_{p} \bar{\ell}} \frac{\hat{R}_{p}}{2\left(i \beta_{p}\right)^{2}} .
$$

In the simple case of a flat upper interface, $u(x) \equiv 0$, we find

$$
R(x)=e^{i \beta_{p}(\bar{u}-\bar{\ell})}\left\{-\left(i \beta_{p}\right) U(x)-\left(i \alpha_{p}\right) \cdot \nabla_{x} \zeta(x)-k^{2} \zeta(x)\right\},
$$

so, since $U=-i \beta_{D} \zeta$,

$$
\hat{R}_{p}=e^{i \beta_{p}(\bar{u}-\bar{\ell})}\left\{\left(i \beta_{p}\right) \hat{U}_{p}-\left(i \alpha_{p}\right) \cdot\left(i \alpha_{p}\right) \hat{\zeta}_{p}+k^{2} \hat{\zeta}_{p}\right\}=e^{i \beta_{p}(\bar{u}-\bar{\ell})}\left\{-2\left(i \beta_{p}\right)^{2}\right\}
$$

and

$$
C_{p}=e^{i \beta_{p} \bar{\ell}} \frac{\hat{R}_{p}}{2\left(i \beta_{p}\right)^{2}}=e^{-i \beta_{p} \bar{\ell}} \frac{1}{2\left(i \beta_{p}\right)^{2}} e^{i \beta_{p}(\bar{u}-\bar{\ell})}\left(-2\left(i \beta_{p}\right)^{2}\right)=-e^{-i \beta_{p} \bar{u}}
$$

\section{$5 \quad$ Numerical Results}

We now present detailed descriptions of numerical simulations conducted with our new approach. As we mentioned above, the scheme is simply Nyström's Method applied to each of the Integral Equations (3.13) which appear in the full layered-medium system (2.5).

\subsection{Exact Solutions}

For non-trivial interface shapes there are no known exact solutions for plane-wave incidence. To establish convergence of our algorithm we utilize the following principle: In building a numerical solver for a homogeneous PDE and boundary conditions:

$$
\begin{array}{ll}
\mathcal{L} u=0 & \text { in } \Omega \\
\mathcal{B} u=0 & \text { at } \partial \Omega,
\end{array}
$$

it is often just as easy to construct an algorithm for the corresponding inhomogeneous problem:

$$
\begin{array}{ll}
\mathcal{L} u=\mathcal{R} & \text { in } \Omega \\
\mathcal{B} u=\mathcal{Q} & \text { at } \partial \Omega
\end{array}
$$

Selecting an arbitrary function $w$, we can compute

$$
\mathcal{R}_{w}:=\mathcal{L} w, \quad \mathcal{Q}_{w}:=\mathcal{B} w,
$$

and now have an exact solution to the problem

$$
\begin{array}{ll}
\mathcal{L} u=\mathcal{R}_{w} & \text { in } \Omega \\
\mathcal{B} u=\mathcal{Q}_{w} & \text { at } \partial \Omega,
\end{array}
$$

namely $u=w$. In this way we can test our inhomogeneous solver for which the homogeneous solver is a special case. However, one should select $w$ which have the same "behavior" as 
solutions $u$ of the homogeneous problem and here we specify $w$ such that $\mathcal{R}_{w} \equiv 0$. We point out though that our exact solution does not correspond to plane-wave incidence (but rather to plane-wave reflection).

To be more specific, consider the functions

$$
v_{r}^{(m)}(x, y)=A^{(m)} e^{i\left(\alpha_{r} \cdot x+\beta_{r}^{(m)} y\right)}+B^{(m)} e^{i\left(\alpha_{r} \cdot x-\beta_{r}^{(m)} y\right)}
$$

with $A^{(M)}=B^{(0)}=0$. These are outgoing, $\alpha$-quasiperiodic solutions of the Helmholtz equation, so that $\mathcal{R}_{w} \equiv 0$ in the notation above. However, the boundary conditions satisfied by these functions are not those satisfied by an incident plane wave. With the construction of the $\mathcal{Q}_{w}$ in mind we compute the surface data

$$
\begin{array}{lll}
\tilde{\zeta}^{(m)}:=v_{r}^{(m-1)}-v_{r}^{(m)} & y=\bar{g}^{(m)}+g^{(m)}(x), & 1 \leq m \leq M \\
\tilde{\psi}^{(m)}:=\partial_{N^{(m)}}\left[v_{r}^{(m-1)}-v_{r}^{(m)}\right] & y=\bar{g}^{(m)}+g^{(m)}(x), & 1 \leq m \leq M .
\end{array}
$$

This is a family of exact solutions against which to test our numerical algorithm for any choice of deformations $\left\{g^{(1)}, \ldots, g^{(M)}\right\}$.

\subsection{Numerical Implementation and Error Measurement}

We utilize Nyström's Method [8] to simulate the Integral Equations (3.13) as they appear in (2.5). In this setting this amounts to enforcing these equations at $N=\left(N_{1}, N_{2}\right)$ equally spaced gridpoints, $x_{j}=\left(x_{1, j_{1}}, x_{2, j_{2}}\right)$, on the period cell $\left[0, d_{1}\right] \times\left[0, d_{2}\right]$, with unknowns being the functions $\left\{\tilde{V}^{(m), l}, V^{(m), l}\right\}$ at these same gridpoints $x_{j}$. The resulting linear system was solved directly in time $\mathcal{O}\left(\left(M N_{x}\right)^{3}\right)$ which sufficed for the purposes of the simulations conducted here.

With these approximations in hand, we can make any number of error measurements versus the exact solutions (5.1). For definiteness we choose to measure the defect in the lower Dirichlet and Neumann traces, and for the results described in $\S 5.3$ we measure

$$
\epsilon_{r e l}:=\sup _{0 \leq m \leq M-1}\left\{\frac{\left|\tilde{V}_{r}^{(m), l}-\tilde{V}_{r}^{(m), l, N}\right|_{L^{\infty}}}{\left|\tilde{V}_{r}^{(m), l}\right|_{L^{\infty}}}, \frac{\left|V_{r}^{(m), l}-V_{r}^{(m), l, N}\right|_{L^{\infty}}}{\left|V_{r}^{(m), l}\right|_{L^{\infty}}}\right\} .
$$

In this, e.g.,

$$
V_{r}^{(m), l}:=v_{r}^{(m)}\left(x, \bar{g}^{(m+1)}+g^{(m+1)}(x)\right), \quad V_{r}^{(m), l, N}:=\sum_{n=0}^{N} V_{r, n}^{(m), l}(x) \varepsilon^{n},
$$

the exact and approximate solutions, respectively.

Remark 5.1. The applicability of our method is determined by the mapping properties of $\mathbf{M}(g)$ and $\mathbf{Q}(g)$, more specifically $\mathbf{M}(g)^{-1}$. Theoretical results along these lines are the subject of our current investigations, but we expect that these properties deteriorate as $g$ becomes more rough (down to Lipschitz $[7,12])$ and as $k^{(m)}$ increases. 


\subsection{Convergence Studies}

For our convergence studies we follow the lead of [19] and select configurations quite close to the ones considered there. To begin we consider the two-dimensional and $2 \pi$-periodic case where the profiles are independent of the $x_{2}$-variable. We will consider the fully three-dimensional case shortly. Recall the three profiles introduced in [21] for precisely this purpose: The sinusoid

$$
f_{s}(x)=\cos (x)
$$

the "rough" $\left(C^{4}\right.$ but not $\left.C^{5}\right)$ profile

$$
f_{r}(x)=\left(2 \times 10^{-4}\right)\left\{x^{4}(2 \pi-x)^{4}-\frac{128 \pi^{8}}{315}\right\},
$$

and the Lipschitz boundary

$$
f_{L}(x)=\left\{\begin{array}{ll}
-(2 / \pi) x+1, & 0 \leq x \leq \pi \\
(2 / \pi) x-3, & \pi \leq x \leq 2 \pi
\end{array} .\right.
$$

We point out that all three profiles have zero mean, approximate amplitude 2, and maximum slope of roughly 1 . The Fourier series representations of $f_{r}$ and $f_{L}$ are listed in [21] and in order to minimize aliasing errors we approximate these by their truncated $P$-term Fourier series, $f_{r, P}$ and $f_{L, P}$.

We begin with two three-layer configurations:

1. (Two Smooth Interfaces, Figure 2) Physical and numerical parameters:

$$
\begin{gathered}
\alpha=0.1, \quad \beta^{(0)}=1.1, \quad \beta^{(1)}=2.2, \quad \beta^{(2)}=3.3 \\
g^{(1)}(x)=\varepsilon f_{s}(x), \quad g^{(2)}(x)=\varepsilon f_{s}(x), \quad \varepsilon=0.01, \quad d=2 \pi, \\
N=10, \ldots, 30 .
\end{gathered}
$$

2. (Rough and Lipschitz Interfaces, Figure 3) Physical and numerical parameters:

$$
\begin{gathered}
\alpha=0.1, \quad \beta^{(0)}=1.1, \quad \beta^{(1)}=2.2, \quad \beta^{(2)}=3.3 \\
g^{(1)}(x)=\varepsilon f_{r, 40}(x), \quad g^{(2)}(x)=\varepsilon f_{L, 40}(x), \quad \varepsilon=0.03, \quad d=2 \pi, \\
N=80, \ldots, 320 .
\end{gathered}
$$

In these three-layer configurations the wavelengths of propagation $\left(\lambda^{(m)}=2 \pi / k^{(m)}\right)$ are

$$
\lambda^{(0)} \approx 5.6885, \quad \lambda^{(1)} \approx 2.8530, \quad \lambda^{(2)} \approx 1.9031 .
$$

In the first configuration, (5.4), we show that only a small number of collocation points $(N \approx 20)$ are required to realize machine precision (up to the conditioning of our algorithm, see Remark 5.2) for small, smooth profiles, (5.3a), which displays the spectral accuracy of the scheme which we anticipate for such profiles based upon our previous results and studies $[20,21,22,23,24]$. In simulation $(5.5)$ we demonstrate that the algorithm performs well if the lower and upper interfaces are replaced by the rough, (5.3b), and Lipschitz, (5.3c), profiles respectively (both truncated after $P=40$ Fourier series terms) provided that $N$ is chosen sufficiently large. 


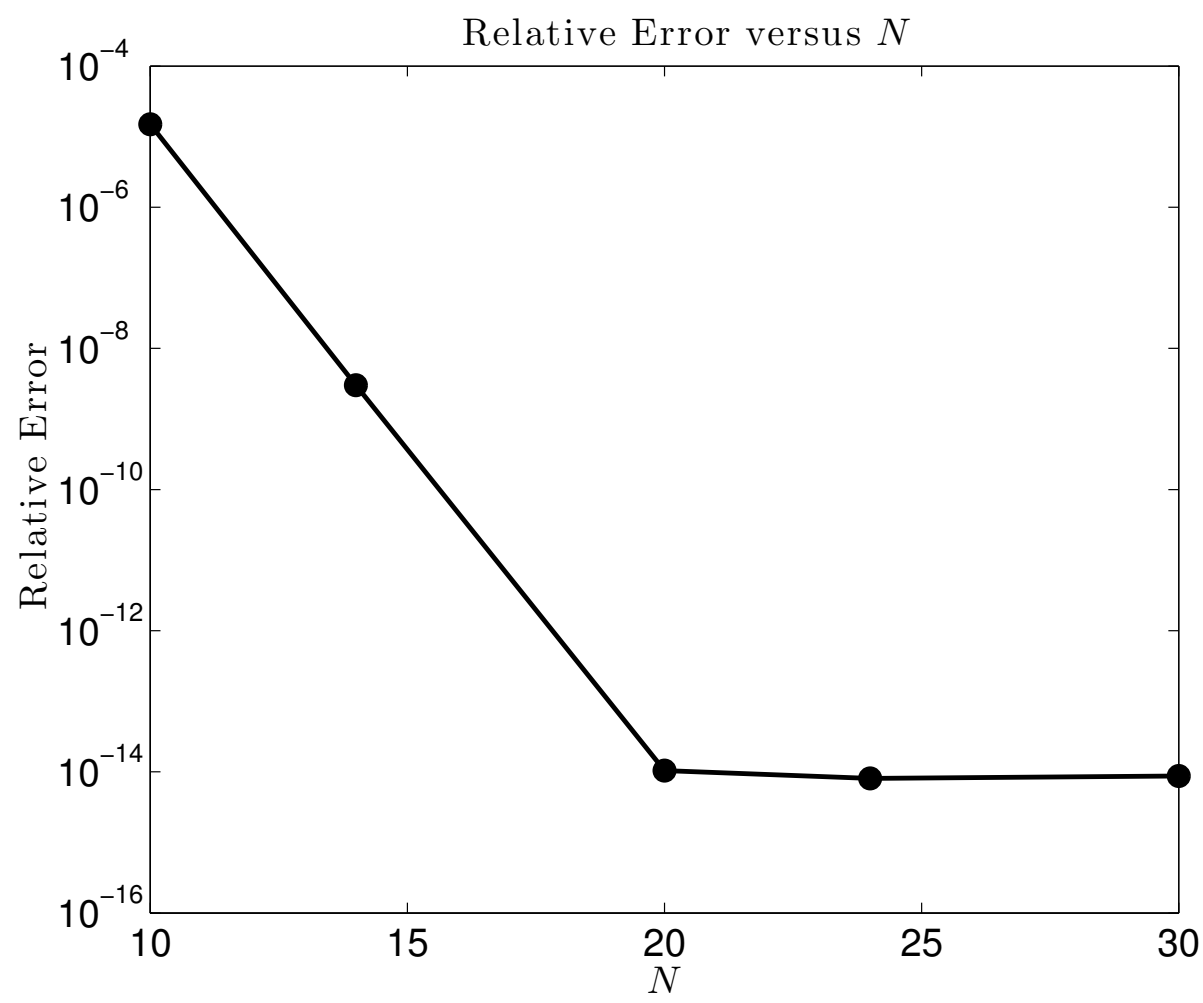

Figure 2: Relative error versus number of gridpoints $N$ for the two-dimensional smoothsmooth configuration, (5.4).

Remark 5.2. Before leaving these first computations we mention the numerical conditioning of our new scheme. Despite their simple form, one can expect the conditioning of the integral operators appearing in (2.5) to degenerate as the profiles in question, $g^{(m)}$, become larger. While the condition numbers for the simulations produced thus far were on the order of $10-100$, if $\varepsilon$ is chosen larger these can grow significantly as we display in Figure 4 . Here we have recomputed the first smooth-smooth configuration, (5.4), for the much larger values of $\varepsilon=0.1,0.15,0.2,0.25$. This necessitates larger choices of $N$ for satisfactory accuracy and so we plot, for $N=20, \ldots, 200$, values of the condition number of $\mathbf{M}$ using MATLAB's cond command [16]. We point out the rather explosive growth of this quantity which illustrates that our method, while extremely simple, fast, and accurate for small to moderately sized deformations, may be inappropriate for "extreme" configurations. We point the interested reader to the preprint of Wilkening \& Vasan [32] for a more detailed investigation in the context of Laplace's equation which arises in the study of surface water waves.

Among the many multilayer configurations our algorithm can address we choose two more, representative ones, in the two-dimensional setting:

1. (Six-Layer, Figure 5) Physical and numerical parameters:

$$
\begin{gathered}
\alpha=0.1, \quad \beta^{(m)}=1.1+m, \quad 0 \leq m \leq 5 \\
g^{(1)}(x)=\varepsilon f_{s}(x), \quad g^{(2)}(x)=\varepsilon f_{r, 40}(x), \quad g^{(3)}(x)=\varepsilon f_{L, 40}(x), \\
g^{(4)}(x)=\varepsilon f_{r, 40}(x), \quad g^{(5)}(x)=\varepsilon f_{s}(x), \quad \varepsilon=0.02, \quad d=2 \pi, \\
N=40, \ldots, 120 .
\end{gathered}
$$




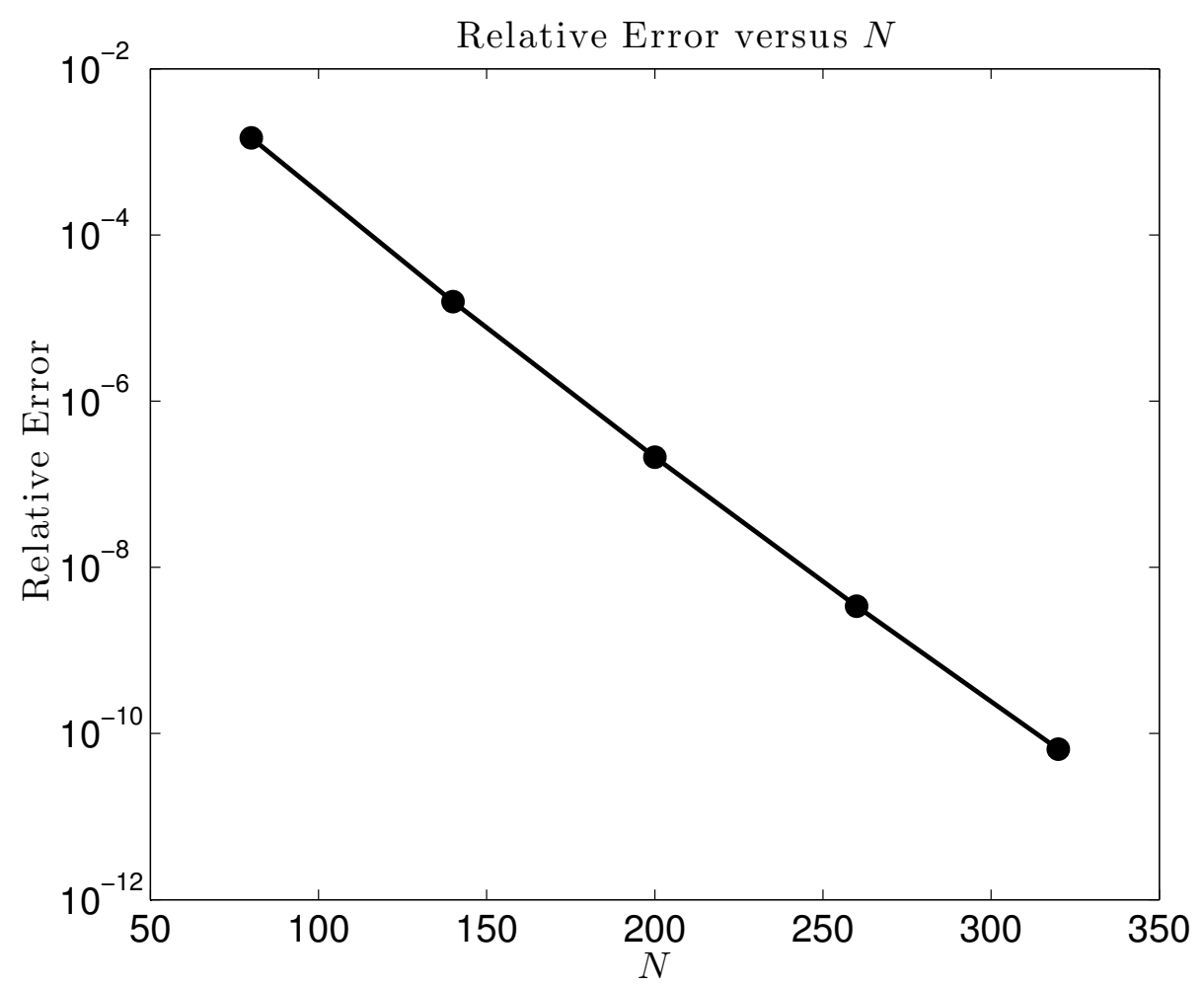

Figure 3: Relative error versus number of gridpoints $N$ for the two-dimensional roughLipschitz configuration, (5.5).

2. (21-Layer, Figure 6) Physical and numerical parameters:

$$
\begin{aligned}
\alpha & =0.1, \quad \beta^{(m)}=\frac{m+1}{10}, \quad 0 \leq m \leq 20 \\
g^{(m)}(x)=\varepsilon f_{s}(x), \quad 1 & \leq m \leq 20, \quad \varepsilon=0.02, \quad d=2 \pi \\
N & =10, \ldots, 30 .
\end{aligned}
$$

Once again, we can see that in all cases, our algorithm provides highly accurate solutions in a stable and rapid manner provided that a sufficient number of degrees of freedom are selected.

We now consider the general case of $(2 \pi) \times(2 \pi)$ periodic interfaces in a three-dimensional structure. Again, we follow [19] and select the following interface shapes: The sinusoid

$$
\tilde{f}_{s}\left(x_{1}, x_{2}\right)=\cos \left(x_{1}+x_{2}\right)
$$

the "rough" $\left(C^{2}\right.$ but not $\left.C^{3}\right)$ profile

$$
\tilde{f}_{r}\left(x_{1}, x_{2}\right)=\left(\frac{2}{9} \times 10^{-3}\right)\left\{x_{1}^{2}\left(2 \pi-x_{1}\right)^{2} x_{2}^{2}\left(2 \pi-x_{2}\right)^{2}-\frac{64 \pi^{8}}{225}\right\},
$$




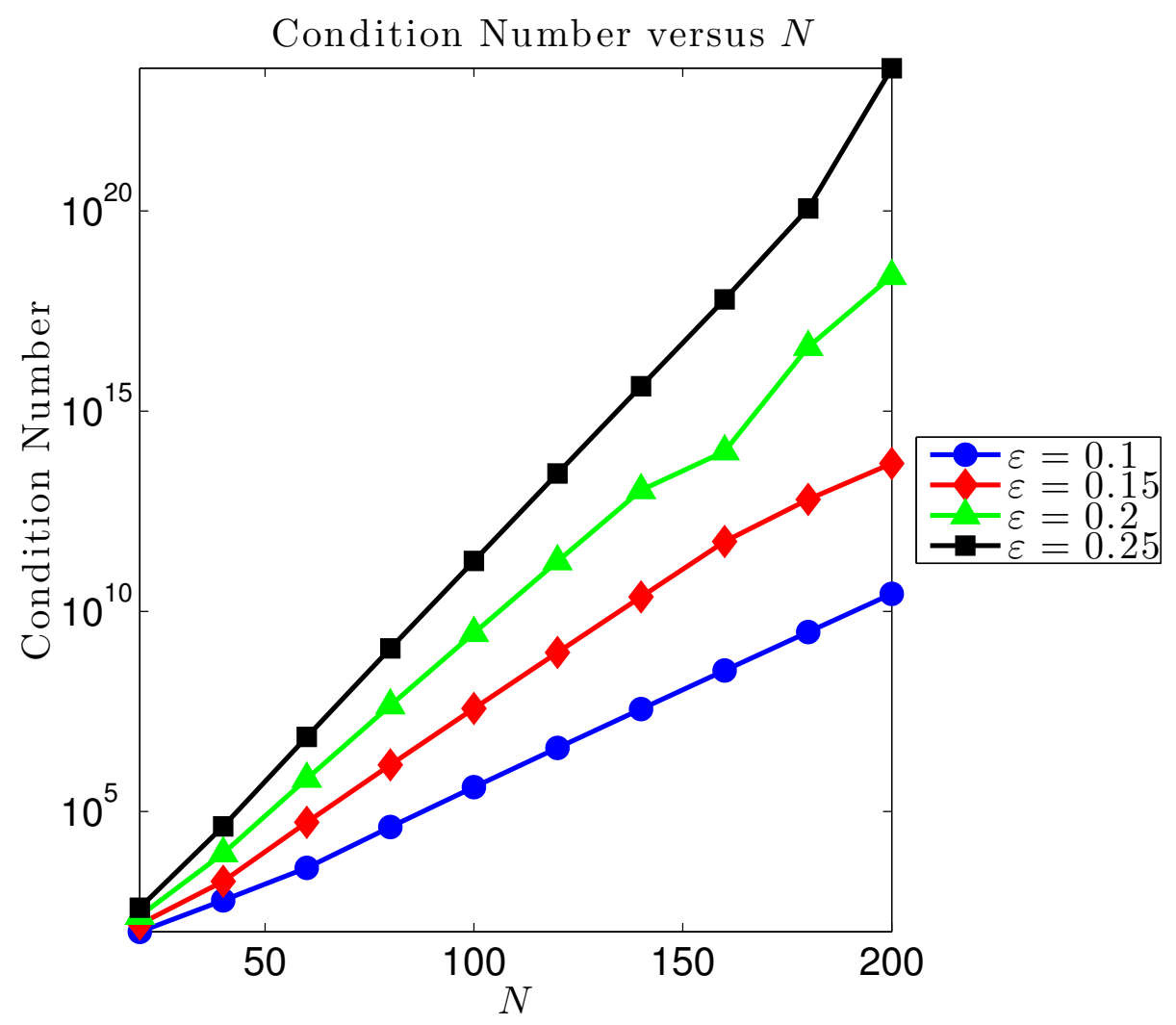

Figure 4: Condition number of $\mathbf{M},(2.5)$, versus number of gridpoints $N$ for the twodimensional smooth-smooth configuration, (5.4).

and the Lipschitz boundary

$$
\tilde{f}_{L}\left(x_{1}, x_{2}\right)=\frac{1}{3}+ \begin{cases}-1+(2 / \pi) x_{1}, & x_{1} \leq x_{2} \leq 2 \pi-x_{1} \\ 3-(2 / \pi) x_{2}, & x_{2}>x_{1}, x_{2}>2 \pi-x_{1} \\ 3-(2 / \pi) x_{1}, & 2 \pi-x_{1}<x_{2}<x_{1} \\ -1+(2 / \pi) x_{2}, & x_{2}<x_{1}, x_{2}<2 \pi-x_{1}\end{cases}
$$

Again, all three profiles have zero mean, approximate amplitude 2, and maximum slope of roughly 1 . The Fourier series representations of $\tilde{f}_{r}$ and $\tilde{f}_{L}$ are given in [21] and in order to minimize aliasing errors we approximate these by their truncation after $P=20$ coefficients, $\tilde{f}_{r, P}$ and $\tilde{f}_{L, P}$.

In three dimensions, despite the ready applicability of our algorithm, the numerical simulations become much more involved. Therefore, we focus upon the two three-layer configurations outlined below.

1. (Two smooth interfaces, Figure 7) Physical and numerical parameters:

$$
\begin{gathered}
\alpha_{1}=0.1, \quad \alpha_{2}=0.2, \quad \beta^{(0)}=1.1, \quad \beta^{(1)}=2.2, \quad \beta^{(2)}=3.3, \\
g^{(1)}\left(x_{1}, x_{2}\right)=\varepsilon \tilde{f}_{s}\left(x_{1}, x_{2}\right), \quad g^{(2)}\left(x_{1}, x_{2}\right)=\varepsilon \tilde{f}_{s}\left(x_{1}, x_{2}\right), \quad \varepsilon=0.1, \\
d_{1}=2 \pi, \quad d_{2}=2 \pi, \quad N_{1}=N_{2}=6, \ldots, 32 .
\end{gathered}
$$




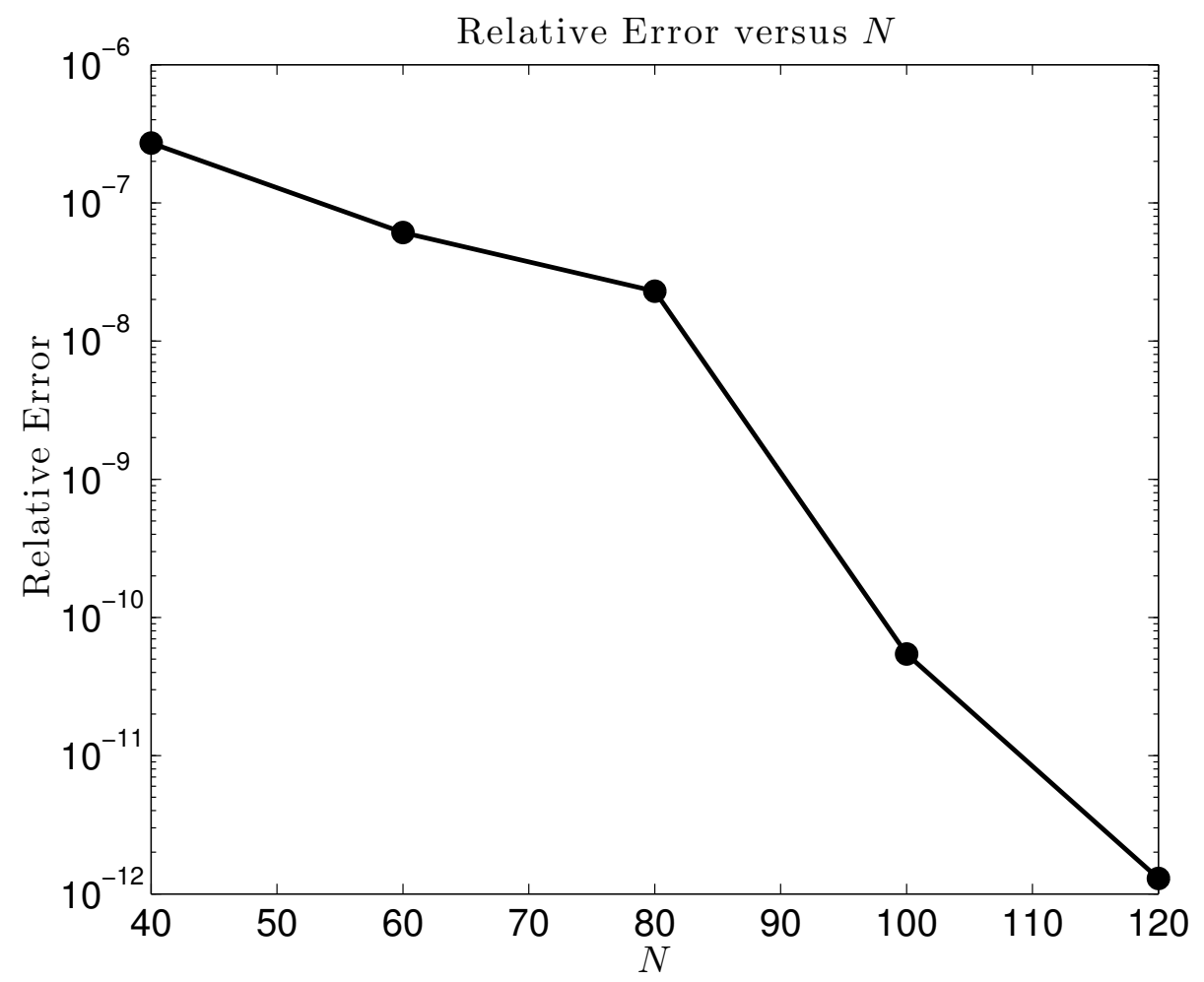

Figure 5: Relative error versus number of gridpoints $N$ for the two-dimensional smoothrough-Lipschitz-rough-smooth configuration, (5.6).

2. (Rough and Lipschitz interfaces, Figure 8) Physical and numerical parameters:

$$
\begin{gathered}
\alpha_{1}=0.1, \quad \alpha_{2}=0.2, \quad \beta^{(0)}=1.1, \quad \beta^{(1)}=2.2, \quad \beta^{(2)}=3.3 \\
g^{(1)}\left(x_{1}, x_{2}\right)=\varepsilon \tilde{f}_{r, 20}\left(x_{1}, x_{2}\right), \quad g^{(2)}\left(x_{1}, x_{2}\right)=\varepsilon \tilde{f}_{L, 20}\left(x_{1}, x_{2}\right), \quad \varepsilon=0.01, \\
d_{1}=2 \pi, \quad d_{2}=2 \pi, \quad N_{1}=N_{2}=8, \ldots, 24 .
\end{gathered}
$$

Again, our algorithm produces highly accurate results in a stable and reliable manner. The behavior is independent of interface shape provided that a sufficient number of collocation points are used.

\subsection{Layered Medium Simulations}

Having verified the validity of our codes, we demonstrate the utility of our approach by simulating plane-wave scattering from all of the configurations described in the previous section. Recall, in two dimensions this included two two-layer (5.4) \& (5.5), and two multiple-layer problems (5.6) \& (5.7); while in three dimensions this featured two twolayer scenarios (5.9) \& (5.10). For this there is no exact solution for comparison so we resort to our diagnostic of energy defect (4.1).

We observe in Figure 9 that we achieve full double precision accuracy with our coarsest discretization for the smooth-smooth configuration, (5.4), while in Figure 10 we show that the same can be realized with $N \approx 200$ for the rough-Lipschitz problem, (5.5). The same generic behavior is noticed for the six-layer configuration, (5.6), and the 21 layer 


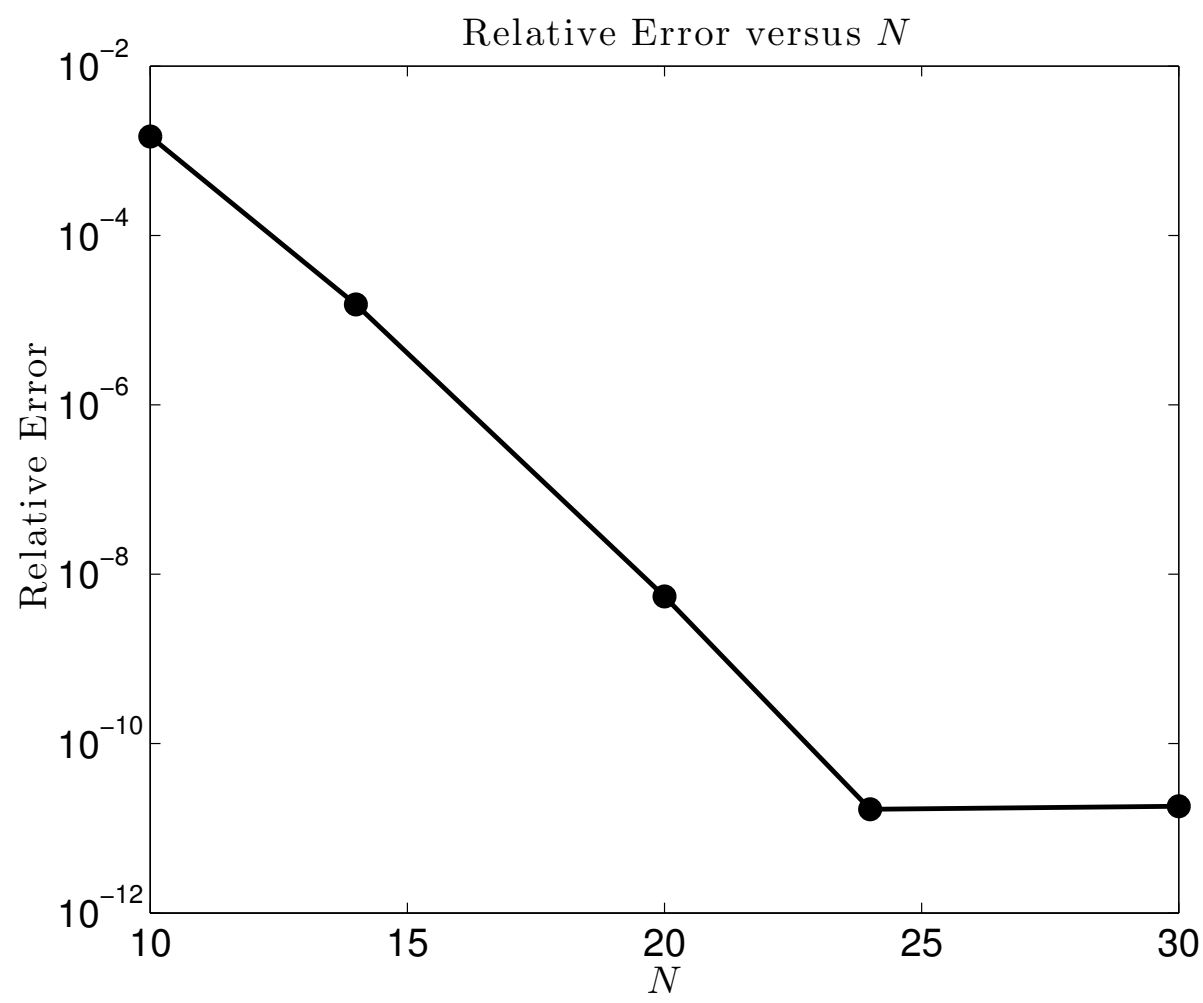

Figure 6: Relative error versus number of gridpoints $N$ for the two-dimensional 21 layer configuration with 20 smooth interfaces, (5.7).

device, (5.7), which are displayed in Figures 11 and 12, respectively. Finally, we display three-dimensional results corresponding to the two-layer problems, (5.9) \& (5.10), and the quantitative results are given in Figures $13 \&$ 14, respectively.

\section{Acknowledgments}

DMA gratefully acknowledges support from the National Science Foundation through grants DMS-1008387 and DMS-1016267. DPN gratefully acknowledges support from the National Science Foundation through grant No. DMS-1115333 and the Department of Energy under Award No. DE-SC0001549.

Disclaimer: This report was prepared as an account of work sponsored by an agency of the United States Government. Neither the United States Government nor any agency thereof, nor any of their employees, make any warranty, express or implied, or assumes any legal liability or responsibility for the accuracy, completeness, or usefulness of any information, apparatus, product, or process disclosed, or represents that its use would not infringe privately owned rights. Reference herein to any specific commercial product, process, or service by trade name, trademark, manufacturer, or otherwise does not necessarily constitute or imply its endorsement, recommendation, or favoring by the United States Government or any agency thereof. The views and opinions of authors expressed herein do not necessarily state or reflect those of the United States Government or any agency thereof. 


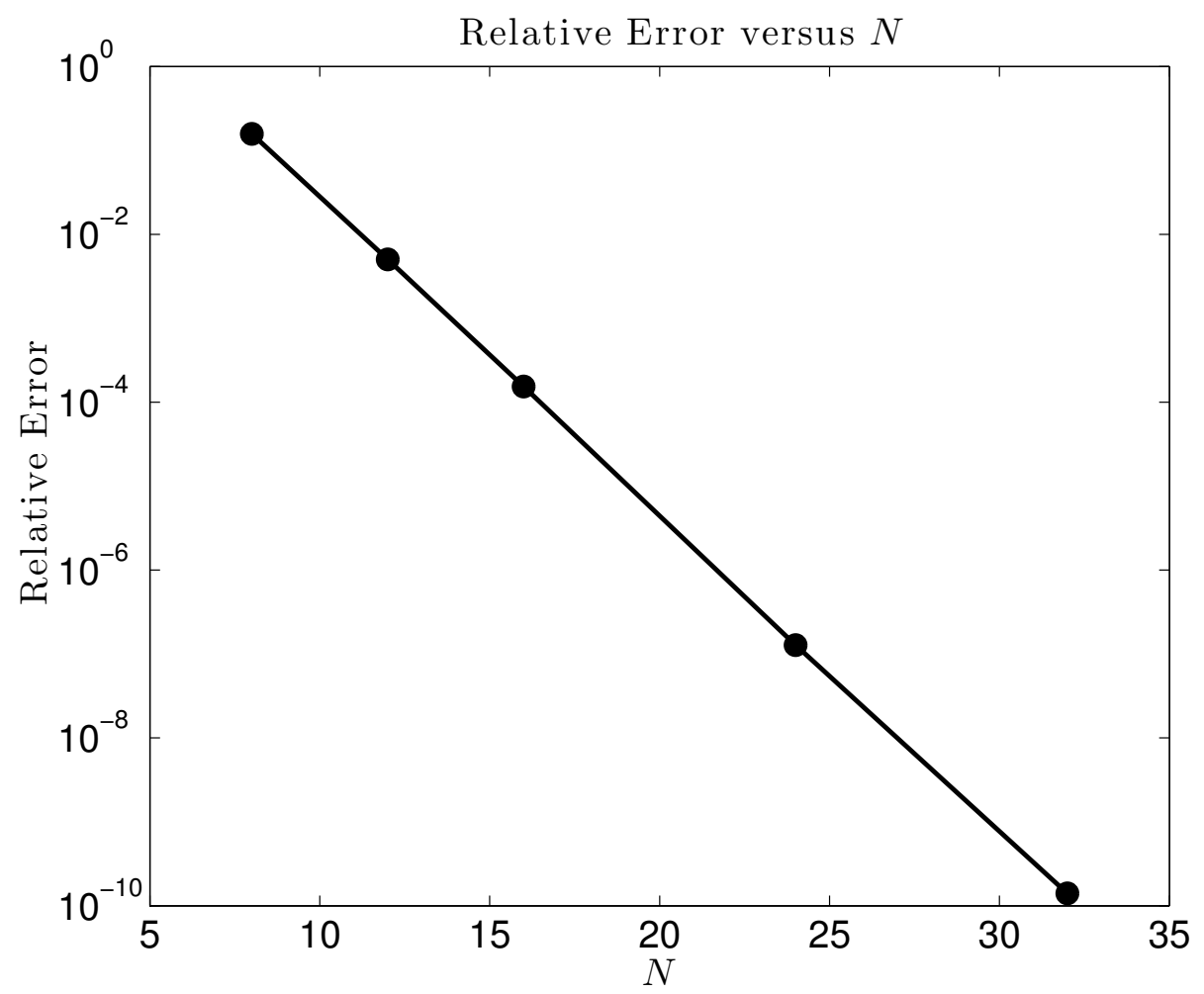

Figure 7: Relative error versus number of gridpoints $N$ for the three-dimensional smoothsmooth configuration, (5.9).

\section{A Alternative Integral Equation Formulations}

The Integral Equations we propose in $\S 3$ are, by no means, the only ones we could consider to approximate solutions of the governing equations (2.5). In fact, if we follow the developments of $\S 3$ we can generate alternatives, and in this appendix we focus on a form for the operator $G$ for the top layer (c.f., $\S 3.1$ ) which we can contrast with (3.5).

To begin, we recall the classical analogue to Fokas' Lemma 3.1 based upon Green's Identity.

Lemma A.1. If we define

$$
Y^{(k)}:=\phi\left(\Delta \psi+k^{2} \psi\right)-\left(\Delta \phi+k^{2} \phi\right) \psi
$$

then

$$
Y^{(k)}=\operatorname{div}_{x}\left[G^{(x)}\right]+\partial_{y}\left[G^{(y)}\right]
$$

where

$$
G^{(x)}:=\phi\left(\nabla_{x} \psi\right)-\left(\nabla_{x} \phi\right) \psi, \quad G^{(y)}:=\phi\left(\partial_{y} \psi\right)-\left(\partial_{y} \phi\right) \psi .
$$

If $\phi$ and $\psi$ solve the Helmholtz equation then $Y^{(k)}=0$, and if $\phi$ is $\alpha$-quasiperiodic and 


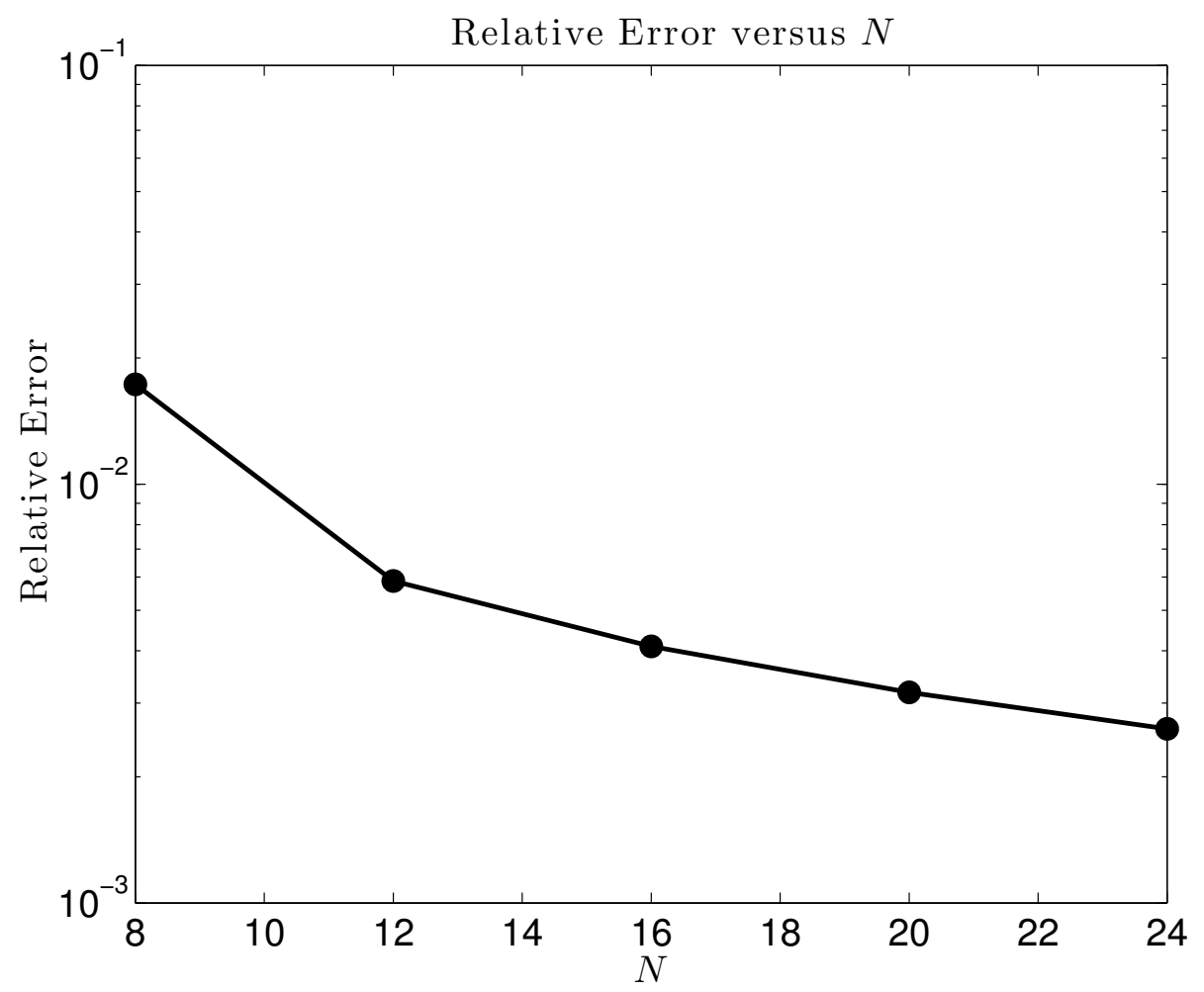

Figure 8: Relative error versus number of gridpoints $N$ for the three-dimensional roughLipschitz configuration, (5.10).

$\psi$ is $(-\alpha)$-quasiperiodic, then the Divergence Theorem gives

$$
\begin{aligned}
0=\int_{\Omega} Y^{(k)} d V=\int_{\partial \Omega} \operatorname{div}[\mathbf{G}] d V=\int_{0}^{d}\left(G^{(x)}\right. & \left.\cdot \nabla_{x} \ell-G^{(y)}\right)_{y=\bar{\ell}+\ell(x)} d x \\
& +\int_{0}^{d}\left(G^{(x)} \cdot\left(-\nabla_{x} u\right)+G^{(y)}\right)_{y=\bar{u}+u(x)} d x .
\end{aligned}
$$

More specifically,

$$
\begin{aligned}
0=\int_{0}^{d}\left[\phi \left(\nabla_{x} \psi\right.\right. & \left.\left.\cdot \nabla_{x} \ell\right)-\nabla_{x} \phi \cdot\left(\psi \nabla_{x} \ell\right)-\phi\left(\partial_{y} \psi\right)+\left(\partial_{y} \phi\right) \psi\right]_{y=\bar{\ell}+\ell(x)} d x \\
& \quad+\int_{0}^{d}\left[(-\phi)\left(\nabla_{x} \psi \cdot \nabla_{x} u\right)+\nabla_{x} \phi \cdot\left(\psi \nabla_{x} u\right)+\phi\left(\partial_{y} \psi\right)-\left(\partial_{y} \phi\right) \psi\right]_{y=\bar{u}+u(x)} d x,
\end{aligned}
$$

and

$$
\begin{aligned}
0=\int_{0}^{d}\left[\phi \left(\nabla_{x} \ell \cdot \nabla_{x} \psi\right.\right. & \left.\left.-\partial_{y} \psi\right)+\psi\left(-\nabla_{x} \ell \cdot \nabla_{x} \phi+\partial_{y} \phi\right)\right]_{y=\bar{\ell}+\ell(x)} d x \\
& +\int_{0}^{d}\left[\phi\left(-\nabla_{x} u \cdot \nabla_{x} \psi+\partial_{y} \psi\right)+\psi\left(\nabla_{x} u \cdot \nabla_{x} \phi-\partial_{y} \phi\right)\right]_{y=\bar{u}+u(x)} d x .
\end{aligned}
$$

Recalling our definitions for $\xi, \zeta, L, U$ from $\S 3$ this becomes

$$
0=\int_{0}^{d} \xi\left(\nabla_{x} \ell \cdot \nabla_{x} \psi-\partial_{y} \psi\right)-\psi L d x+\int_{0}^{d} \zeta\left(-\nabla_{x} u \cdot \nabla_{x} \psi+\partial_{y} \psi\right)-\psi U d x
$$




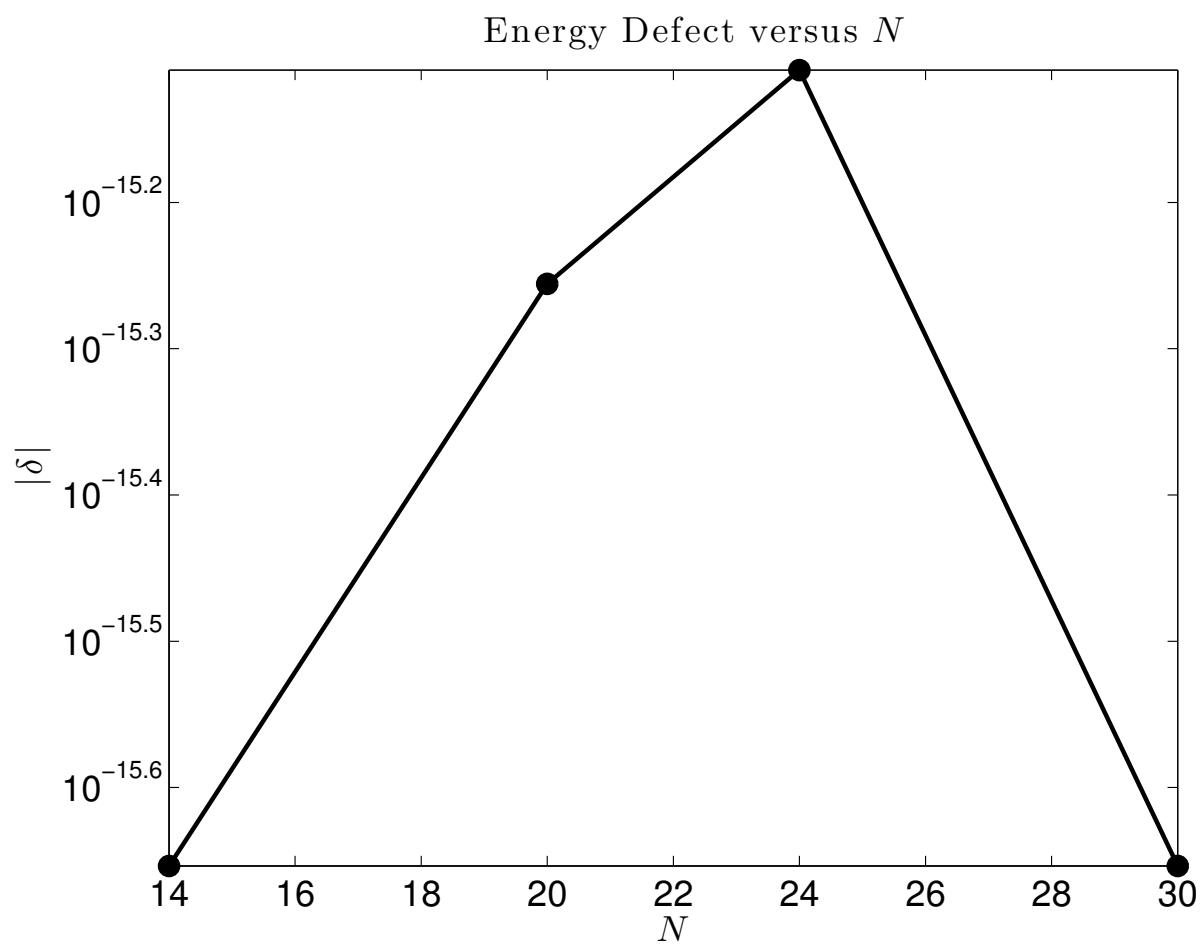

Figure 9: Energy defect versus number of gridpoints $N$ for the two-dimensional smoothsmooth configuration, (5.4).

or

$$
\begin{aligned}
\int_{0}^{d} \psi U d x+\int_{0}^{d} \psi L d x=\int_{0}^{d}\left(-\nabla_{x} u \cdot \nabla_{x} \psi+\partial_{y} \psi\right) \zeta & d x \\
& +\int_{0}^{d}\left(\nabla_{x} \ell \cdot \nabla_{x} \psi-\partial_{y} \psi\right) \xi d x .
\end{aligned}
$$

To produce a DNO for the top layer we set $u(x) \equiv 0$, and if we choose

$$
\psi(x, y)=e^{-i \alpha_{p} \cdot x+i \beta_{p}(y-\bar{\ell})},
$$

then the terms at $y=\bar{u}$ cancel. With this choice (A.1) becomes

$$
\int_{0}^{d} \psi L d x=\int_{0}^{d}\left(\nabla_{x} \ell \cdot \nabla_{x} \psi-\partial_{y} \psi\right) \xi d x
$$

and with the $\psi$ defined above

$$
\int_{0}^{d} e^{i \beta_{p} \ell} e^{-i \alpha_{p} \cdot x} L d x=\int_{0}^{d} e^{i \beta_{p} \ell} e^{-i \alpha_{p} \cdot x}\left(\nabla_{x} \ell \cdot\left(-i \alpha_{p}\right)-i \beta_{p}\right) \xi d x .
$$

Once again, renaming the DNO $G$ and the interface $g$ we discover

$$
\int_{0}^{d} e^{i \beta_{p} g} e^{-i \alpha_{p} \cdot x} G d x=\int_{0}^{d} e^{i \beta_{p} g} e^{-i \alpha_{p} \cdot x}\left(\nabla_{x} g \cdot\left(-i \alpha_{p}\right)-i \beta_{p}\right) \xi d x,
$$




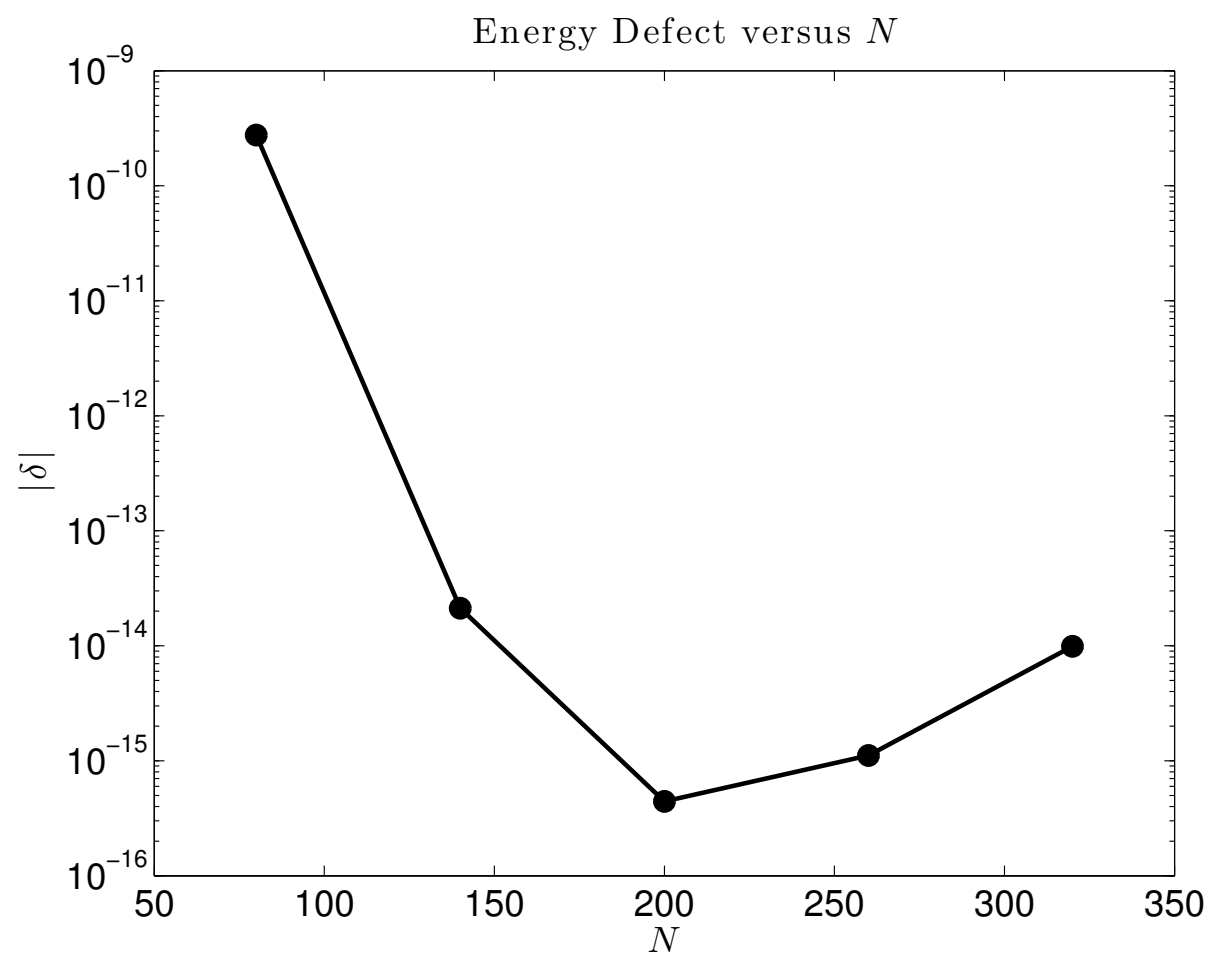

Figure 10: Energy defect versus number of gridpoints $N$ for the two-dimensional roughLipschitz configuration, (5.5).

which should be compared with (3.5). In particular, we note the explicit appearance of the derivative of $g$ in (A.2).

One can ask if there is a simple relationship between these two formulations, and, in fact, there is. We recall (3.5), divided by $\left(i \beta_{p}\right)$,

$$
\int_{0}^{d} e^{i \beta_{p} g} e^{-i \alpha_{p} x} G d x=\int_{0}^{d}\left(\frac{i \alpha_{p}}{i \beta_{p}}\right) e^{i \beta_{p} g} e^{-i \alpha_{p} x} \cdot \nabla_{x} \xi d x+\int_{0}^{d}\left(\frac{k^{2}}{i \beta_{p}}\right) e^{i \beta_{p} g} e^{-i \alpha_{p} x} \xi d x,
$$

and define

$$
I=\int_{0}^{d}\left(\frac{i \alpha_{p}}{i \beta_{p}}\right) e^{i \beta_{p} g} e^{-i \alpha_{p} x} \cdot \nabla_{x} \xi d x
$$

We integrate by parts (using the periodicity of the integrand)

$$
\begin{aligned}
I & =-\int_{0}^{d} \operatorname{div}_{x}\left[\left(\frac{i \alpha_{p}}{i \beta_{p}}\right) e^{i \beta_{p} g} e^{-i \alpha_{p} \cdot x}\right] \xi d x \\
& =\int_{0}^{d}\left\{-\left(i \alpha_{p}\right) \cdot \nabla_{x} g+\left(\frac{\left|i \alpha_{p}\right|^{2}}{i \beta_{p}}\right)\right\} e^{i \beta_{p} g} e^{-i \alpha_{p} \cdot x} \xi d x,
\end{aligned}
$$

so that

$$
\int_{0}^{d} e^{i \beta_{p} g} e^{-i \alpha_{p} x} G d x=\int_{0}^{d}\left\{-\left(i \alpha_{p}\right) \cdot \nabla_{x} g+\left(\frac{\left|i \alpha_{p}\right|^{2}}{i \beta_{p}}\right)+\left(\frac{k^{2}}{i \beta_{p}}\right)\right\} e^{i \beta_{p} g} e^{-i \alpha_{p} x} \xi d x .
$$




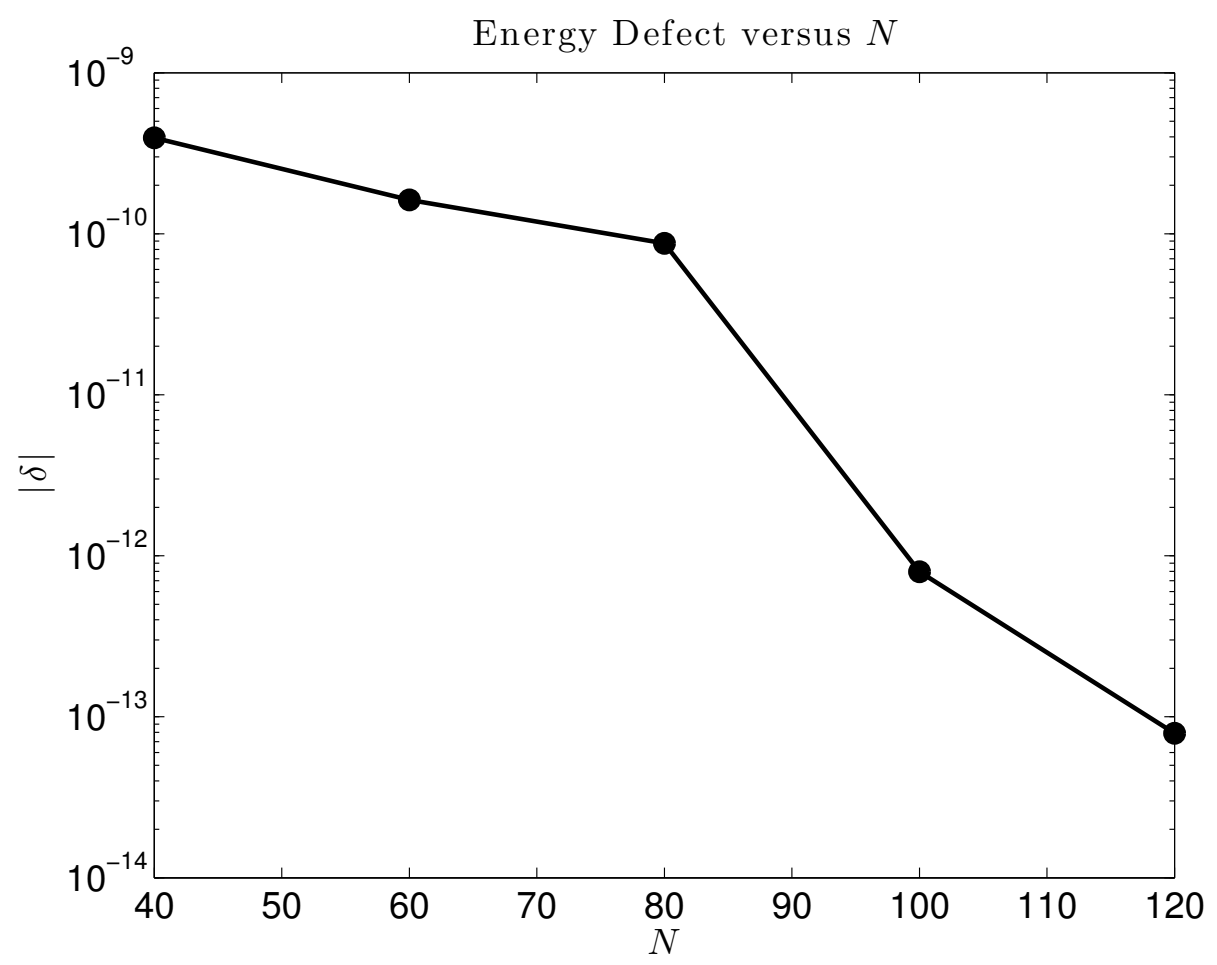

Figure 11: Energy defect versus number of gridpoints $N$ for the two-dimensional smoothrough-Lipschitz-rough-smooth configuration, (5.6).

Using the fact that $\left|i \alpha_{p}\right|^{2}+k^{2}=-\left(i \beta_{p}\right)^{2}$

$$
\int_{0}^{d} e^{i \beta_{p} g} e^{-i \alpha_{p} x} G d x=\int_{0}^{d} e^{i \beta_{p} g} e^{-i \alpha_{p} x}\left\{-\left(i \alpha_{p}\right) \cdot \nabla_{x} g-\left(i \beta_{p}\right)\right\} \xi d x,
$$

which is (A.2). Thus the two are equivalent up to an integration by parts.

\section{References}

[1] M. J. Ablowitz, A. S. Fokas, and Z. H. Musslimani. On a new non-local formulation of water waves. J. Fluid Mech., 562:313-343, 2006.

[2] L. M. Brekhovskikh and Y. P. Lysanov. Fundamentals of Ocean Acoustics. SpringerVerlag, Berlin, 1982.

[3] Oscar P. Bruno and Fernando Reitich. Numerical solution of diffraction problems: A method of variation of boundaries. J. Opt. Soc. Am. A, 10(6):1168-1175, 1993.

[4] Oscar P. Bruno and Fernando Reitich. Numerical solution of diffraction problems: A method of variation of boundaries. II. Finitely conducting gratings, Padé approximants, and singularities. J. Opt. Soc. Am. A, 10(11):2307-2316, 1993.

[5] Oscar P. Bruno and Fernando Reitich. Numerical solution of diffraction problems: A method of variation of boundaries. III. Doubly periodic gratings. J. Opt. Soc. Am. A, 10(12):2551-2562, 1993. 


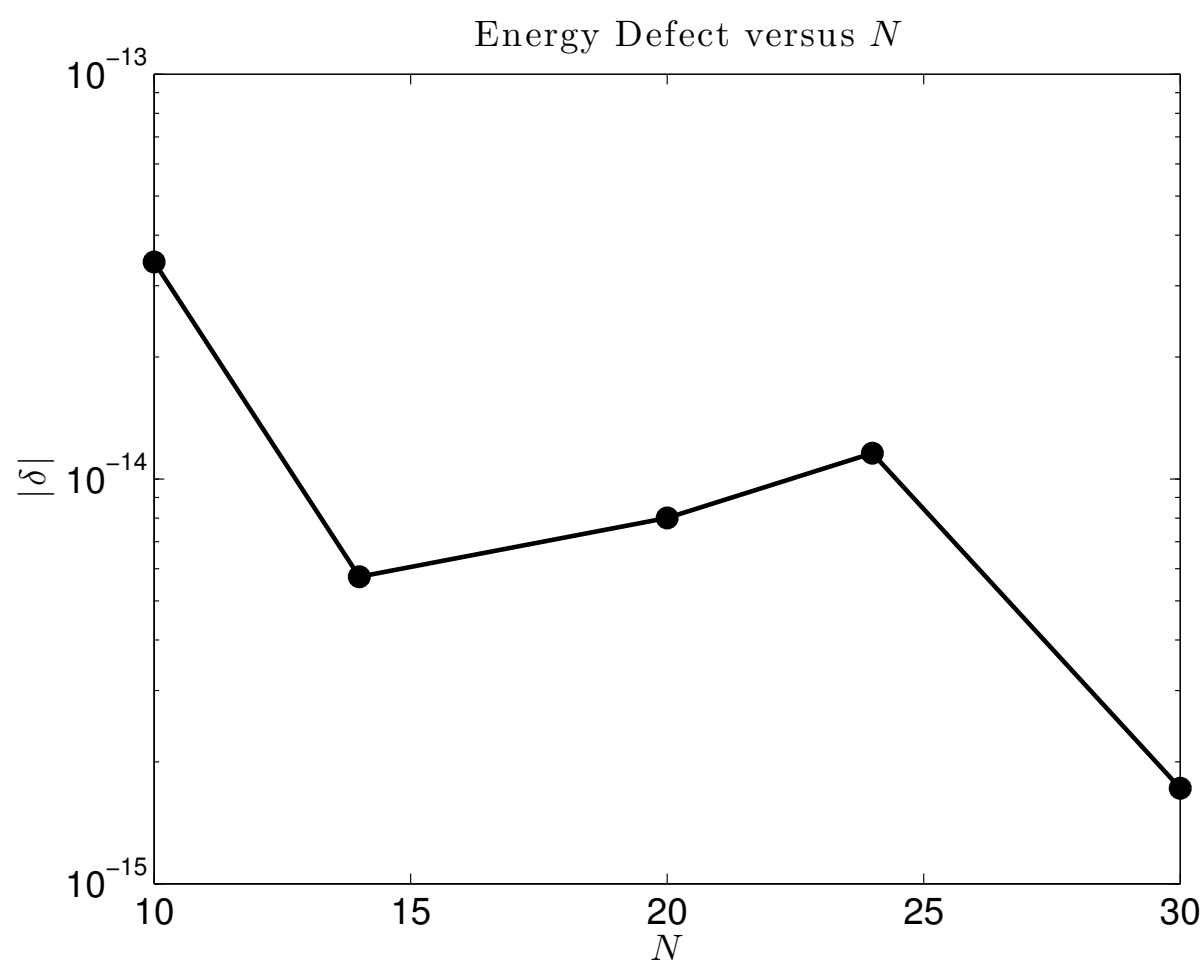

Figure 12: Energy defect versus number of gridpoints $N$ for the two-dimensional 21 layer configuration with 20 smooth interfaces, (5.7).

[6] R. Coifman, M. Goldberg, T. Hrycak, M. Israeli, and V. Rokhlin. An improved operator expansion algorithm for direct and inverse scattering computations. Waves Random Media, 9(3):441-457, 1999.

[7] R. Coifman and Y. Meyer. Nonlinear harmonic analysis and analytic dependence. In Pseudodifferential operators and applications (Notre Dame, Ind., 1984), pages 71-78. Amer. Math. Soc., 1985.

[8] David Colton and Rainer Kress. Inverse acoustic and electromagnetic scattering theory. Springer-Verlag, Berlin, second edition, 1998.

[9] Athanassios S. Fokas. A unified approach to boundary value problems, volume 78 of CBMS-NSF Regional Conference Series in Applied Mathematics. Society for Industrial and Applied Mathematics (SIAM), Philadelphia, PA, 2008.

[10] David Gottlieb and Steven A. Orszag. Numerical analysis of spectral methods: theory and applications. Society for Industrial and Applied Mathematics, Philadelphia, Pa., 1977. CBMS-NSF Regional Conference Series in Applied Mathematics, No. 26.

[11] L. Greengard and V. Rokhlin. A fast algorithm for particle simulations. J. Comput. Phys., 73(2):325-348, 1987.

[12] Bei Hu and David P. Nicholls. Analyticity of Dirichlet-Neumann operators on Hölder and Lipschitz domains. SIAM J. Math. Anal., 37(1):302-320, 2005. 


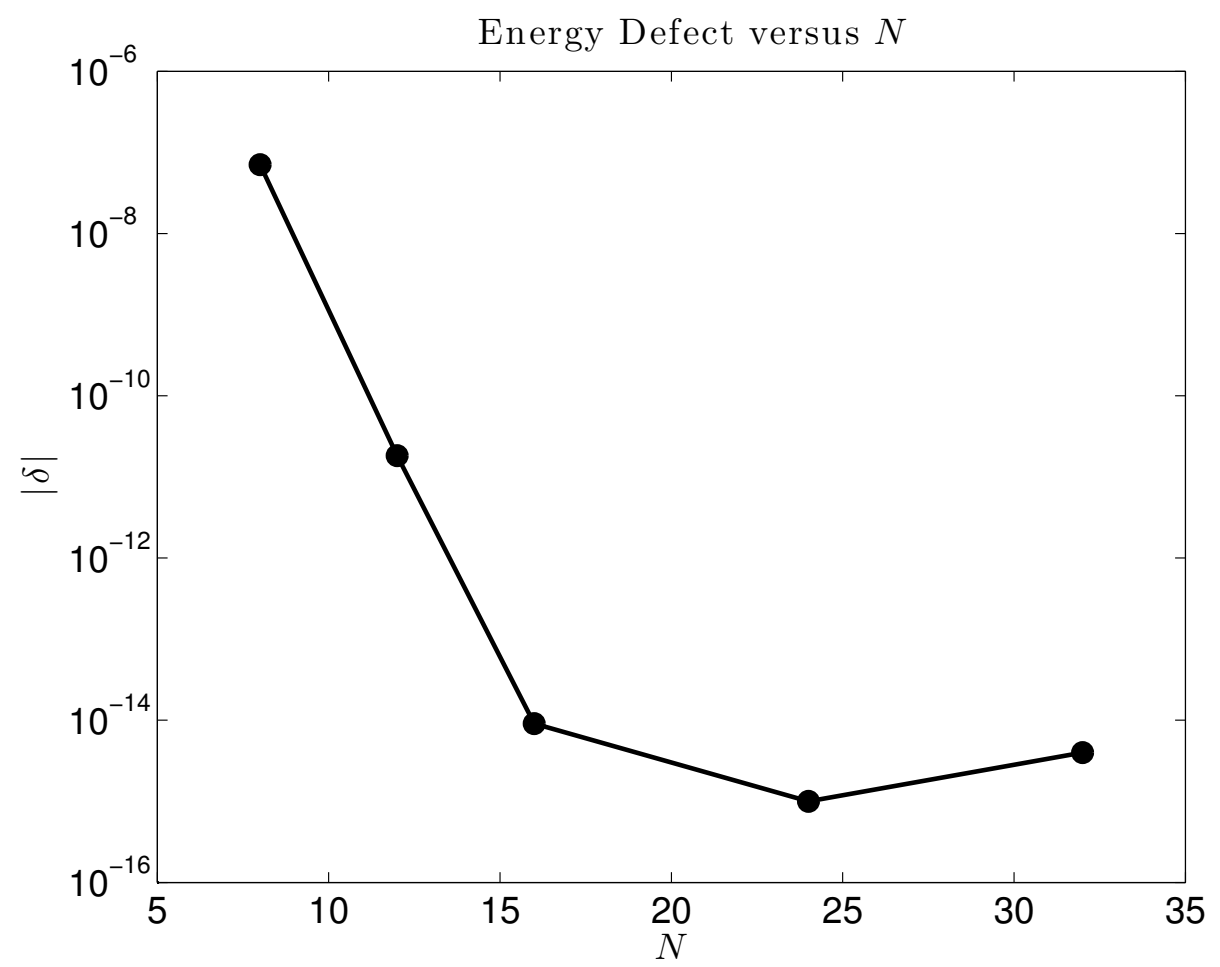

Figure 13: Energy defect versus number of gridpoints $N$ for the three-dimensional smoothsmooth configuration, (5.9).

[13] D. Komatitsch and J. Tromp. Spectral-element simulations of global seismic wave propagation-I. Validation. Geophysical Journal International, 149(2):390-412, 2002.

[14] Alison Malcolm and David P. Nicholls. A boundary perturbation method for recovering interface shapes in layered media. Inverse Problems, 27(9):095009, 2011.

[15] Alison Malcolm and David P. Nicholls. A field expansions method for scattering by periodic multilayered media. Journal of the Acoustical Society of America, 129(4):17831793, 2011.

[16] MATLAB. version 7.10 .0 (R2010a). The MathWorks Inc., Natick, Massachusetts, 2010 .

[17] D. Michael Milder. An improved formalism for rough-surface scattering of acoustic and electromagnetic waves. In Proceedings of SPIE - The International Society for Optical Engineering (San Diego, 1991), volume 1558, pages 213-221. Int. Soc. for Optical Engineering, Bellingham, WA, 1991.

[18] D. Michael Milder. An improved formalism for wave scattering from rough surfaces. J. Acoust. Soc. Am., 89(2):529-541, 1991.

[19] David P. Nicholls. Three-dimensional acoustic scattering by layered media: A novel surface formulation with operator expansions implementation. Proceedings of the Royal Society of London, A, 468:731-758, 2012. 


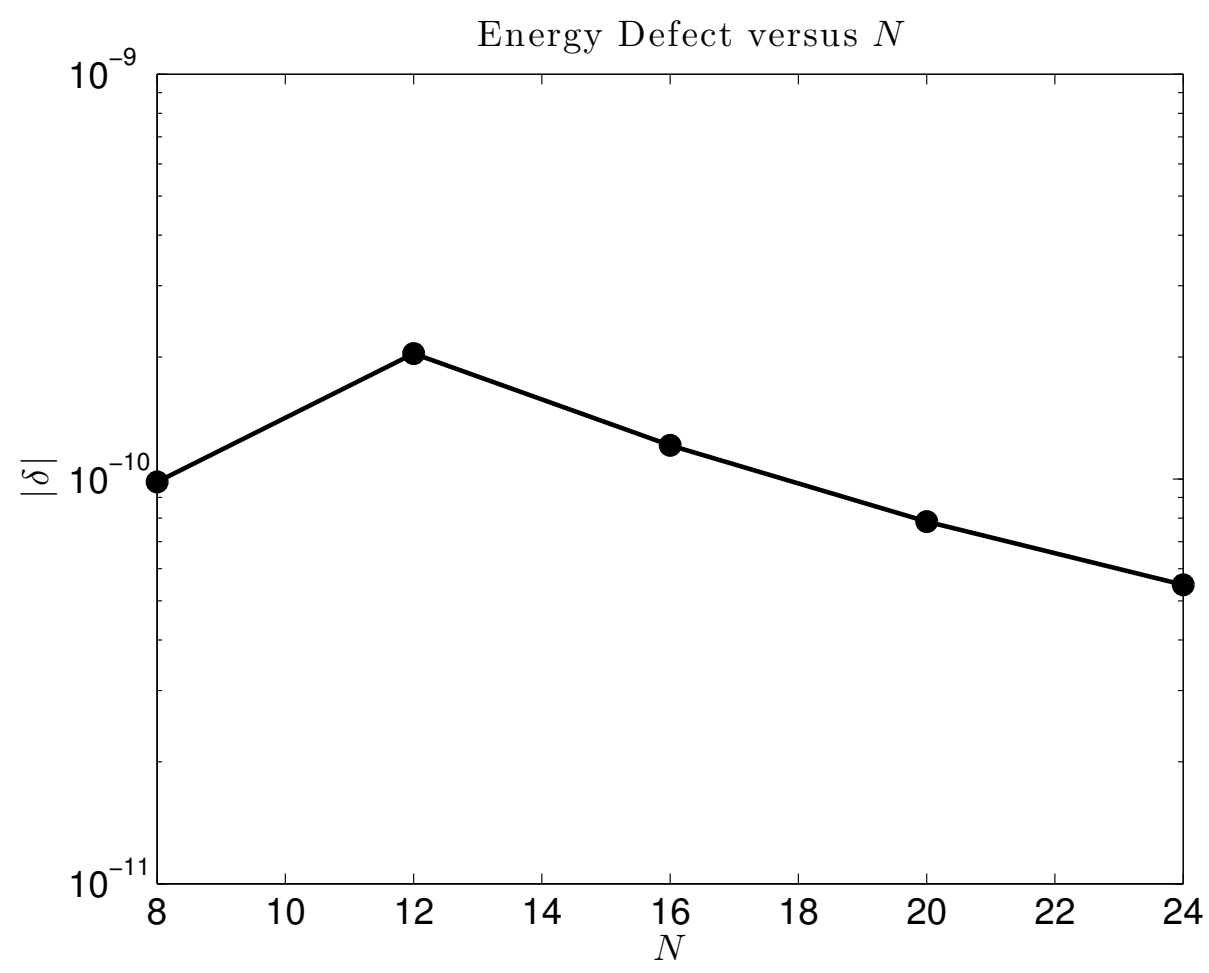

Figure 14: Energy defect versus number of gridpoints $N$ for the three-dimensional roughLipschitz configuration, (5.10).

[20] David P. Nicholls and Fernando Reitich. A new approach to analyticity of DirichletNeumann operators. Proc. Roy. Soc. Edinburgh Sect. A, 131(6):1411-1433, 2001.

[21] David P. Nicholls and Fernando Reitich. Stability of high-order perturbative methods for the computation of Dirichlet-Neumann operators. J. Comput. Phys., 170(1):276$298,2001$.

[22] David P. Nicholls and Fernando Reitich. Analytic continuation of Dirichlet-Neumann operators. Numer. Math., 94(1):107-146, 2003.

[23] David P. Nicholls and Fernando Reitich. Shape deformations in rough surface scattering: Cancellations, conditioning, and convergence. J. Opt. Soc. Am. A, 21(4):590-605, 2004.

[24] David P. Nicholls and Fernando Reitich. Shape deformations in rough surface scattering: Improved algorithms. J. Opt. Soc. Am. A, 21(4):606-621, 2004.

[25] Roger Petit, editor. Electromagnetic theory of gratings. Springer-Verlag, Berlin, 1980.

[26] R. Gerhard Pratt. Frequency-domain elastic wave modeling by finite differences: A tool for crosshole seismic imaging. Geophysics, 55(5):626-632, 1990.

[27] F. Reitich and K. Tamma. State-of-the-art, trends, and directions in computational electromagnetics. CMES Comput. Model. Eng. Sci., 5(4):287-294, 2004. 
[28] FJ Sanchez-Sesma, E Perez-Rocha, and S Chavez-Perez. Diffraction of elastic waves by three-dimensional surface irregularities. part II. Bulletin of the Seismological Society of America, 79(1):101-112, 1989.

[29] E. A. Spence and A. S. Fokas. A new transform method I: domain-dependent fundamental solutions and integral representations. Proc. R. Soc. Lond. Ser. A Math. Phys. Eng. Sci., 466(2120):2259-2281, 2010.

[30] E. A. Spence and A. S. Fokas. A new transform method II: the global relation and boundary-value problems in polar coordinates. Proc. R. Soc. Lond. Ser. A Math. Phys. Eng. Sci., 466(2120):2283-2307, 2010.

[31] L. Tsang, J. A. Kong, and R. T. Shin. Theory of Microwave Remote Sensing. Wiley, New York, 1985.

[32] J. Wilkening and V. Vasan. Comparison of four popular methods of computing the Dirichlet-Neumann operator for the water-wave problem. submitted, 2014.

[33] O. C. Zienkiewicz. The Finite Element Method in Engineering Science, 3rd ed. McGraw-Hill, New York, 1977. 